\title{
Resolution of inflammation: a new therapeutic frontier
}

James N Fullerton and Derek W Gilroy

Centre for Clinical Pharmacology and Therapeutics, Division of Medicine, 5 University Street, University College London, London WC1E 6JJ, UK.

d.gilroy@ucl.ac.uk.

\section{ABSTRACT}

INTRODUCTION

ACUTE INFLAMMATION, RESOLUTION AND POST-RESOLUTION

ANTI-INFLAMMATION VERSUS PRO-RESOLUTION

CHRONIC INFLAMMATION AND "FRUSTRATED RESOLUTION"

INITIATION OF RESOLUTION

1) Removal of stimuli

2) Dampening pro-inflammatory signalling

3) Pro-inflammatory catabolism

RESOLUTION

1) Leukocyte death

2) Clearance of dead cells

MACROPHAGE PHENOTYPES DURING RESOLUTION

TARGETING PRO-RESOLUTION PATHWAYS

FUTURE STRATEGIES TO TARGET RESOLUTION

CONCLUSIONS AND FUTURE DIRECTIONS

BOX 1: PMNS in adaptive immunity

BOX 2: Alternative forms of cell death

BOX 3: Challenges with translation into humans

BOX 4: Murine and zebrafish models of inflammation-resolution

TABLE 1: Efforts to target resolution pathways

GLOSSARY OF TERMINOLOGY USED 


\begin{abstract}
Dysregulated inflammation is a central pathological process in diverse disease states. Traditionally, therapeutic approaches have sought to modulate the pro- or anti-inflammatory limbs of inflammation, with mixed success. However, insight into the pathways by which inflammation is resolved has highlighted novel opportunities to pharmacologically manipulate these processes, which might represent a complementary (and perhaps even superior) therapeutic approach. This review discusses the state of the art in the biology of resolution of inflammation, highlighting the opportunities and challenges for translational research in this field.
\end{abstract}

\title{
Introduction
}

The past 20 years have afforded greater clarity about Celsus' four cardinal signs of inflammation - namely, calor (heat), dolor (pain), rubor (redness) and tumor (swelling) - and how they might be reversed or resolved. Arising from these advances, it has been proposed that some diseases that are associated with chronic inflammation may be underpinned by dysregulated resolution as much as driven by ongoing pro-inflammatory processes, and that therapy based on rectifying these defects will help guide ongoing inflammation down a pro-resolution pathway..

Pro-resolution strategies intrinsically afford greater scope than conventional antiinflammatory approaches; however, despite our burgeoning understanding of resolution biology, clear limitations remain. Pharmacologically tackling the fifth cardinal sign - functio laesa (loss of tissue function) - is challenging. Equally, just as inflammation onset follows many diverse courses, resolution pathways are heterogeneous, with evidence to date suggesting they are probably tissue- and stimulus-specific. Thus, although central cellular and molecular mediators may be elucidated, it is unclear whether any one pro-resolution regimen will act as a panacea for multiple disease states.

In this Review, we provide an update on the field of inflammation and resolution, describing the key cellular players, and the factors that regulate their phenotype, fate and clearance. We then describe recent evidence that effective resolution represents a bridge between innate and adaptive immunity. Moreover, we introduce the idea that diseases characterized by chronic inflammation may be associated with 'frustrated resolution', whereby the inability to adequately resolve acute inflammation leads to maladaptive immunity. Finally, we highlight efforts to target pro-resolution pathways in diseases involving inflammation and models in which such strategies are investigated. We wish to provoke a re-think on how chronic inflammation is addressed therapeutically; arguing that the development of novel pro-resolving agents may be as beneficial as, and perhaps synergistic with or superior to, the administration of anti-inflammatory drugs.

\section{Normal and 'frustrated' resolution}

Recent evidence indicates that, following normal inflammation and resolution, a third phase known as post-resolution occurs, in which the affected tissue develops adaptive immunity. Here, we briefly describe inflammation, normal resolution and post-resolution, and suggest that in certain situations chronic inflammation may 
represent a case of 'frustrated resolution' where the goal of achieving adaptive immunity is not met.

Inflammation. Inflammation is a protective reaction of the microcirculation, initiated after infection and/or injury. Local and systemic inflammatory responses both aim to eliminate the inciting stimulus, promote tissue repair and healing and, in the case of infection, establish immune memory such that the host mounts a faster and more specific response on a future encounter.

The acute inflammatory response is a complex yet highly coordinated sequence of events involving molecular, cellular and physiological alterations. It begins with the production of soluble mediators (including complement; chemokines; cytokines; eicosanoids, such as prostaglandins; free radicals; and vasoactive amines) by resident cells - (that is, tissue macrophages, dendritic cells (DCs), lymphocytes, endothelial cells, fibroblasts and mast cells) in the injured or infected tissue. Concomitantly, cell adhesion molecules are upregulated on circulating leukocytes and endothelial cells, promoting promote the exudation of proteins and influx of granulocytes from the blood. This well-characterized phase of the inflammatory response is routinely targeted using drugs such as non-steroidal anti-inflammatory drugs (NSAIDs) and pro-inflammatory cytokine-negating biologics (for example, tumour necrosis factor (TNF)-specific antibodies) that inhibit or antagonise the action of these mediators, and currently form the mainstay for the treatment of chronic inflammatory disease.

Upon arrival, these leukocytes (typically polymorphonuclear cells (PMNs) in the case of non-specific inflammation or eosinophils in response to allergens) primarily function to phagocytose and eliminate tissue debris and microorganisms through distinct intracellular mechanisms (for example, involving superoxide radicals, myeloperoxidase, proteases and lactoferrins) and/or extracellular mechanisms (such as neutrophil extracellular traps). The type of effector cells, number of cells and ability of these effector cells to neutralize the inciting stimuli probably signal the next phase of pro-resolution processes.

Resolution. The period between peak inflammatory cell influx and their clearance from the tissue site and restoration of functional homeostasis - that is, classically defined resolution - is no longer considered a passive event during which inflammation simply fizzles out ${ }^{1,2}$. Instead, a complex, tightly regulated, cascade of processes occur. First, the injurious agents that triggered the inflammatory response are eliminated. Subsequently, the synthesis of pro-inflammatory mediators is suspended, and any such mediators that remain are catabolised, stopping further leukocyte recruitment and oedema formation. Next, regardless of whether the initial response was PMN- or eosinophil-driven or adaptive to re-call antigens (lymphocyte mediated), immune cells are cleared from the tissue. Inflammatory leukocytes can re-enter systemic circulation, but many influxed PMNs, eosinophils and lymphocytes undergo local apoptosis or necrosis and subsequent efferocytosis by recruited monocyte-derived macrophages (MDMs). Multiple intricate signalling mechanisms and factors control each of these processes and the balance between them, including cell-to-cell receptor binding and humoral mediators, particularly bioactive lipids. Once efferocytosis is complete, macrophages can leave the inflamed site by 
lymphatic drainage; however, evidence also suggests that a small population may undergo local apoptosis ${ }^{3}$.

Post-resolution. Recently, we demonstrated that classical resolution may not be the end of the local immune response; rather, there is a third phase that we termed postresolution ${ }^{4}$. Traditionally, resolution processes were deemed successful if acute inflammation was terminated; however they may have a hitherto unappreciated role in controlling adaptive immune responses and maintaining tolerance. Specifically, we found that following the resolution of murine innate-immune responses to a low dose of Saccharomyces cerevisiae cell wall extract (zymosan; administered intraperitoneally (i.p.)) or ovalbumin-labelled Streptococcus pneumoniae (also i.p.), there was a previously overlooked second influx of leukocytes into tissues that persisted for weeks (Fig. 1a). These cells comprised three separate populations of

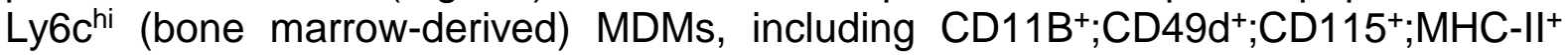
myeloid-derived suppressor cells (MDSCs); $\mathrm{F} 480^{\mathrm{lo}} ; \mathrm{MHC}-\mathrm{II}^{+} ; \mathrm{CD}^{+} 1 \mathrm{C}^{+} \mathrm{DCs}$; and

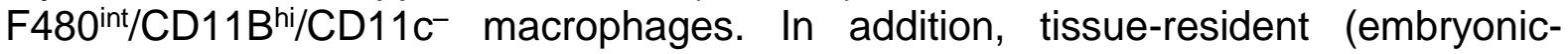
derived) macrophages, which disappeared during the acute inflammatory response, re-appeared. These diverse populations of macrophages were observed alongside lymph node expansion and increased numbers of memory $T$ and $B$ lymphocytes in the peripheral blood and tissue. PMNs were not observed in the affected tissues during this phase. Based on our studies and the work of others ${ }^{5-8}$, we concluded that DCs residing in naive tissues take up antigens and migrate to local lymph nodes to stimulate the initial steps in T-cell activation. As inflammation resolves, however, we found that Ly6c ${ }^{\text {hi }}$ monocyte-derived macrophages and/or DCs - either from the resolving site or from peripheral blood - further amplify the adaptive arm of the response while tissue-resident macrophages preferentially phagocytose apoptotic $\mathrm{PMNs}^{4,9}$. These Ly6c ${ }^{\text {hi }}$ monocyte derived macrophages remain in tissues for months after inflammation has resolved, and help to dictate the magnitude and duration of subsequent acute innate inflammatory responses ${ }^{4,10}$.

As these observations were not seen when resolution was disrupted (Fig. 1b), we suggest that local pro-resolution processes help bridge innate and adaptive immunity ${ }^{4}$ (Fig. 1a,2). Thus, resolution could be redefined as the creation of a tissue microenvironment that facilitates interaction between the innate and adaptive arms of the immune system ${ }^{4}$. Indeed, examples exist to support this idea: for one, inflammatory adjuvants typically aid vaccine efficacy ${ }^{11}$. Traditionally, adjuvants were thought to act as a depot from which the antigen was slowly released, enhancing antibody production. Now, however, they are believed primarily to trigger an acute inflammatory reaction, thereby recruiting and activating antigen-presenting cells that capture the antigen. Additionally, adjuvants are believed to possibly help convert soluble antigens into a particulate form so that they are easily phagocytosed by antigen-presenting cells. The process of bridging innate and adaptive immunity also involves PMNs, a function elaborated in Box 1.

Chronic inflammation and 'frustrated resolution'. If resolution is a bridge between innate and adaptive immunity, chronic inflammation could be explained as reflecting incomplete resolution of the initial acute response that, in turn, does not fully engage an appropriate adaptive immune response. 
This idea is supported by evidence that blocking the persistent type I interferon (IFN) response to lymphocytic choriomeningitis virus reduces innate immune activation, restores lymph node architecture and results in enhanced $\mathrm{CD}^{+} \mathrm{T}$ cell-mediated clearance of the virus ${ }^{12,13}$. It emerges that whereas early, acute production of type 1 IFNs promotes virus clearance, chronic exposure to these IFNs triggers immunosuppression via interleukin-10 (IL-10), programmed cell death ligand 1 (PDL1) and indolamine signalling, and causes T cell apoptosis, collectively impairing the host's ability to develop specific immunity ${ }^{14,15}$. Similarly, chronic exposure to TNF attenuates $T$ cell or adaptive immune responses. Repeated injection of TNF in mice dampens $T$ cell responsiveness to recall antigens, whereas chronic anti-TNF therapy enhances T-cell reactivity ${ }^{16}$. Thus, whereas many early-phase Th-1-type cytokines drive inflammation, their persistence may paradoxically impair resolution, resulting in reduced antigen clearance and prevention of the development of adaptive immunity or long-term immune memory (Fig. 1b). Conceptually, many diseases driven by chronic inflammation may involve ongoing acute inflammation alongside frustrated attempts to develop adaptive immunity, with the latter ultimately turning maladaptive. (Fig. 1).

\section{Anti-inflammation versus pro-resolution.}

The idea that resolution processes cause not only the termination of inflammation but also assist the shift from innate to adaptive immunity affords additional opportunity to target diseases that involve inflammation or chronic inflammation specifically, by promoting resolution. It has therefore been particularly important to dissociate the meaning of 'anti-inflammation' from that of 'pro-resolution'1, 17. Many investigators confuse the two concepts by reporting on the use of, for example, prophylactic (that is, pre-initiating stimuli) pharmacological inhibition, targeted inhibition of factors expressed during early inflammatory onset, or the use of mice that over or underexpress genes that are implicated in both inflammation and resolution ${ }^{18-22}$. Targeting only resolution should involve modifying the course of an already established inflammation-driven disease, in a clinically relevant manner, by harnessing endogenous 'off switches' (be they signalling cascades or cellular interactions) that cause inflammation to resolve (Fig. 2).

Many effective anti-inflammatory treatment regimens are on the market (including NSAIDs, anti-cytokine therapies and steroids); however, switching off the underlying disease process is much more challenging. Hypothetically, diseases that are driven by ongoing inflammation could be treated by activating pro-resolution pathways that might be pathologically silenced and/or by agonising functional pro-resolution pathways. Indeed, combining pro-resolution with anti-inflammatory technology might transpire to be a superior strategy to existing approaches. However, there is circumstantial evidence from human studies that inflammation and resolution may not be mutually exclusive events. For instance, although macrophages are the principle drivers of synovial inflammation in patients with rheumatoid arthritis, the immediate decrease in the numbers of cells in the synovial fluid observed after beginning the anti-inflammatory TNF-specific antibody therapy was not due to apoptosis at the site of inflammation ${ }^{23}$. Given the steady infiltration of monocytes into the synovium in rheumatoid arthritis ${ }^{24}$, the rapid decrease in synovial macrophages 
observed after antirheumatic treatment might result from an alteration in macrophage retention, rather than changes in monocyte influx. Thus, this disease might be sensitive to treatments targeting the synovial retention of inflammatory cells - in which case, simply inhibiting ongoing inflammation may promote resolution (see below).

Hastening resolution was originally feared to lead to incomplete clearance of the original trigger, especially in the context of infections; however, specialised proresolution mediators have now been shown not just to promote resolution processes, but also to augment cellular anti-microbial function, for instance increasing PMN and macrophage bacterial phagocytosis ${ }^{25}$. Thus, unlike anti-inflammatory approaches, approaches to augment pro-resolution pathways should not increase susceptibility to infection per se.

\section{Initiation of resolution}

Generally, conventional anti-inflammatory therapies are based upon the inhibition of factors that drive inflammation - that is, they antagonise pro-inflammation signals. Although this has afforded huge success in certain fields (such as rheumatoid arthritis and inflammatory bowel disease) there remains scope for improvement. Insights into resolution biology have afforded the possibility of harnessing factors that catabolise pro-inflammatory signalling; inhibiting pathways that break down proresolution mediators; or selectively and potently activating receptors upon which proresolution mediators act. The processes described below that lead to resolution and the factors that control them are not tied to any disease or tissue, but recount, in general terms, advances made in resolution biology.

Removal of stimuli. For resolution to occur effectively the initiating injurious agents must be eliminated. This is particularly relevant for autoimmune diseases such as rheumatoid arthritis and systemic lupus erythematosus (SLE) that are driven by intractable endogenous antigens 26,27 . In the case of bacterial infection, the removal of the pathogen is facilitated in part by NADPH oxidase-mediated killing by $\mathrm{PMNs}^{28}$. Defects in the assembly of NADPH subunits, as occurs in the immunodeficient disorder chronic granulomatous disease and in tuberculosis, demonstrate the importance of antigen clearance for resolution ${ }^{29}$.

Dampening pro-inflammatory signalling. Following stimulus removal, it is rational to suggest that the various families of receptors and their signalling pathways that triggered the release of pro-inflammatory mediators be turned off in order to prevent collateral damage from an ongoing pro-inflammatory state. Sepsis is a good example of when this does not occur: a cytokine storm may be witnessed despite negative bacterial blood cultures, suggesting exaggerated and persistent activation of the innate immune system despite removal of the stimulus. A number of regulatory mechanisms have evolved to keep the signalling factors that drive the innate immune system in check $^{30}$.

For example, the transmembrane molecule leucine rich repeat-containing protein 33 (LRRC33) negatively regulates Toll-like receptor (TLR) signaling and subsequent nuclear factor-KB (NF-KB) activation, thus dampening TLR-driven pro-inflammatory events $^{31}$. LRRC33 is functionally similar to RP105 (also known as CD180), an 
endogenous inhibitor of TLR4 signaling., DCs lacking RP105 exhibit increased NF$\mathrm{KB}$ activation and pro-inflammatory signaling in response to lipopolysaccharide (LPS) challenge ${ }^{32}$. Separately, the multifunctional deubiquitinase cylindromatosis was recently shown to negatively regulate NF-KB signalling and to be an inducible negative-feedback regulator of bacteria-induced inflammation ${ }^{33}$. Additionally, the immune-suppressive lipid mediator prostaglandin E2 $\left(\mathrm{PGE}_{2}\right)^{34}$ inhibits protein expression of TLR4 ${ }^{35}$. Taken together, mediators such as LRRC33, RP105, cylindromatosis and $\mathrm{PGE}_{2}$ typically ensure that inflammatory signalling is dampened after acute inflammatory responses.

Post-transcriptional regulatory mechanisms - such as those that restrict the stability and/or translation of various mRNAs - have also evolved. Many of these mRNAs possess adenine- or uridine-rich elements (AREs) that recruit destabilizing factors and translational silencers to their $3^{\prime}$ untranslated regions (UTRs) ${ }^{36}$. ARE-binding proteins that destabilize these mRNAs include tristetraprolin (TTP), B-related factor 1 (BRF1), BRF2, KH type-splicing regulatory protein (KSRP; also known as FUBP2), and AU-rich element RNA-binding protein 1 (AUF1). TTP interacts with AREs via its zinc finger and destabilizes mRNA transcripts that encode several inflammatory modulators, including granulocyte-macrophage colony-stimulating factor (GM$\mathrm{CSF})^{37}, \mathrm{CSF}^{25}, \quad \mathrm{IL}-2^{38}, \quad \mathrm{IL}-6^{39}$, inducible nitric oxide synthase $(\mathrm{iNOS})^{40}$, cyclooxygenase $2(\mathrm{COX} 2)^{41}$, IFNy ${ }^{42}$ and TNF ${ }^{43}$. Transcription of TTP itself is also triggered in a feedback loop by inflammatory-like stimuli including TNF ${ }^{43}$, LPS ${ }^{44}$, and IFNy ${ }^{39}$, in order to dampen the expression of inflammatory cytokines and promote resolution of inflammation ${ }^{43}$. By contrast, ARE-binding proteins that inhibit mRNA translation include $T$ cell intracellular antigen 1 (TIA1), TIA receptor (TIAR), CUG triplet repeat RNA-binding protein 2 (CUGBP2; also known as CELF2) and fragile Xrelated protein 1 (FXR1) ${ }^{45}, 46$. Targeted deletion of Tiar enhances the production of pro-inflammatory proteins in LPS-activated macrophages, and mice lacking TIA1 are more susceptible to LPS-induced septic shock than wild-type littermates, owing to overproduction of $\mathrm{TNF}^{47}$. The factors described here to regulate pro-inflammatory signalling at the mRNA level therefore represent alternative targets for the development of novel drugs to treat diseases driven by on-going or over exuberant inflammation.

Steroid receptor co-activator 3 (SRC3) is a component of complexes that involve NF$\mathrm{KB}$, peroxisome proliferator-activated receptor (PPAR) and glucocorticoid receptor transcription factor. Interestingly, SRC3 can either promote or inhibit inflammatory responses by regulating rates of mRNA transcription. $\mathrm{SrC}^{-1-}$ mice are hypersensitive to LPS challenge because they overexpress pro-inflammatory cytokines such as TNF, IL-6 and IL-1 $\beta$. Specifically, the translation of Tnf and II1 b transcripts is higher in macrophages lacking SRC3 than in wild-type controls. Indeed, SRC3 binds TIA1, leading to an increase in the binding of TIA1 to the ARE of Tnf mRNA, inhibiting its translation. Thus, SRC3 and TIA1 cooperatively repress the expression of reporter constructs that bear the TNF 3' UTR. Together these findings show that SRC3 functions as both a transcriptional co-activator and a translational co-repressor. This is an example of a regulatory protein that is involved in both the initiation and resolution phases of the inflammatory response, thereby representing a possible opportunity to increase or reduce inflammation as clinically appropriate. 
IFNy-treated macrophages provide another example of a temporally staggered negative-feedback inhibition of cell activation ${ }^{48}$. IFNy induces the expression of the acute-phase response protein ceruloplasmin ${ }^{48,49}$ as well as the assembly of the IFNy-activated inhibitor of translation (GAIT) complex, which binds to the GAIT element. The GAIT element is a 29-nucleotide hairpin found in the 3' UTRs of ceruloplasmin mRNA, which, in turn, targets components of the translation initiation machinery to inhibit protein synthesis ${ }^{50}$. As a result, after ceruloplasmin is secreted from IFNy-activated macrophages in order to combat infection or injury, its synthesis is turned off. Transcripts encoding several other inflammatory mediators - including CCL22, CCR3, CCR4 and CCR6 - contain functional GAIT elements ${ }^{51,52}$, suggesting the GAIT complex has an important influence on the magnitude and duration of inflammatory reactions.

The transcription factor zinc finger $\mathrm{CCCH}$ domain-containing protein $12 \mathrm{~A}(\mathrm{ZC} 3 \mathrm{H} 12 \mathrm{~A})$ acts downstream of CCL2, and is another immunoregulatory zinc-finger protein that negatively regulates macrophage activation and TNF and iNOS production; it does this by inhibiting NF-KB ${ }^{53}$. Indeed, production of IL-6 and IL-12p40, but not of TNF, is increased in TLR-activated ZC3H12A-deficient macrophages owing to increased I/6 and I/12p40 mRNA stability. In addition, ZC3H12A-deficient mice succumb to a chronic inflammatory condition that is characterized by increased levels of immunoglobulins and autoantibodies, increased lung-infiltrated leukocytes and hyperresponsive T cells and macrophages ${ }^{54}$.

Several microRNAS (miRNAs) have emerged as key regulators of TLR proinflammatory signalling pathways. For example, the NF-kB-inducible miRNA miR146 inhibits translation of mRNA encoding IRAK1, IRAK2 and TRAF6 ${ }^{55}$. As IRAK1 and TRAF6 are required for NF-KB activation, this function of miR-146 leads to a negative regulatory loop ${ }^{56}$. By corollary, overexpression of miR-146 results in a decrease in various chemokines and cytokines, including CXCL8, CCL5 ${ }^{56}$, IL-6, CXCL8 $8^{57,58}$ and IL-1 $1 \beta^{59}$, and thereby prevents overinflammation, bringing the system back to homeostasis. As expected, mir-146a-knockout mice exhibit loss of immunological tolerance and their macrophages are hyperresponsive to LPS. Another miR, miR-21, is induced in macrophages via NF-KB and MyD88 upon LPS stimulation. miR-21 controls inflammation by downregulating the translation of the pro-inflammatory tumor suppressor programmed cell death 4 (PD4) ${ }^{60}$, an inhibitor of IL-10 production. As IL-10 blocks NF-KB, miR-21 represents another negative feedback regulator of LPS-driven inflammation, with potential in the resolution of inflammation.

Elucidating the role of negative feedback regulators of inflammation is an attractive strategy. Indeed, miRNAs are increasingly popular drug targets. Proteins that are considered difficult to modulate pharmacologically can now be targeted via their miRNA gene regulators, enabling the treatment of currently seemingly untractable diseases. Targeting miRNAs requires innovation at the level of drug discovery; this is well described elsewhere (see Ref. ${ }^{61}$ ).

However, not all miRNAs or ARE-binding proteins dampen pro-inflammatory signalling pathways. For example, miR-15562, 54 enhances the expression of TNF in macrophages and ZCCHC11 stabilises mRNA that encodes IL-6 ${ }^{64}$. Nonetheless, defects in one or more ARE-binding proteins and/or miRNAs could explain the 
aetiology of some ongoing inflammation-driven diseases, such as sepsis. Although there is more study of anti-inflammatory signalling pathways (such as cyclic AMP, IL10 and transforming growth factor- $\beta 1$ (TGF $\beta 1)$ ) than of the negative regulation of pro-inflammatory signalling pathways, the latter nonetheless represents a critically important aspect of biology and medicine. Moreover, this area of research provides an opportunity to pharmacologically manipulate their function in order to switch off persistent inflammation at its source.

Clearance of pro-inflammatory mediators. Following disposal of the injurious agent and turning off signalling pathways, the levels of cytokines, chemokines, eicosanoids, cell adhesion molecules and other inflammatory mediators must revert back to their pre-inflamed state.

One example of a type of pro-inflammatory mediator that is catabolised during resolution is the prostaglandins, which are involved in vasodilation, oedema formation and allodynia (reviewed in detail in Ref. ${ }^{65}$ ). Prostaglandins first undergo oxidation of the 15(S)-hydroxyl group by a 15-hydroxyprostaglandin dehydrogenase (which metabolizes E-series prostaglandins, lipoxins, 15-HETE and 5,15-diHETE to their corresponding 15-keto compounds). Second, an NADPH- or NADH-dependent $\Delta 13-15$-ketoprostaglandin reductase reduces the $\Delta 13$ double bond. Further catabolism of prostaglandins, some HETES and lipoxins occurs via the betaoxidation pathway, which is common to fatty acids in general. Specifically, the carboxyl end of the molecule is catabolised to form short-chain metabolites that are excreted in the urine. Some of the eicosanoids are also excreted following glucuronidation. 5-HETE and leukotrienes (LTS) undergo beta-oxidation from the omega-terminus following an initial omega-hydroxylation. Disruption of these pathways is known to contribute to disease states (for example, sepsis) and targeted augmentation may be expected to reverse pathological alterations ${ }^{66}$.

Atypical chemokine receptors such as D6 bind ligands without initiating classical signalling pathways, thereby acting as a type of scavenging system for proinflammatory signals. D6-deficient mice produce an excess of chemokines in response to 12-O-tetradecanoylphorbol-13-acetate (TPA)-induced skin inflammation, resulting in a notable inflammatory pathology with similarities to human psoriasis (for review see Ref. ${ }^{67}$ ). In addition, the chemokines CCL3 and CCL5 are increased in peritoneal exudates of $\mathrm{Ccr5}^{-/}$mice during the resolution of acute peritonitis, but in these animals are scavenged by apoptotic PMNs in a CCR5-dependent manner. CCR5 expression on the surface of apoptotic PMNs was reduced by proinflammatory cytokines and increased by pharmacological application of proresolution lipid mediators such as lipoxin $\mathrm{A}_{4}{ }^{68}$. Thus, there are endogenous systems that can facilitate the clearance of pro-inflammatory mediators and that, when dysregulated, may lead to chronic inflammation. Conversely, pro-inflammatory catabolic pathways could be harnessed therapeutically to drive ongoing inflammation towards resolution.

\section{Resolution}

Cell death and the clearance of effete leukocytes is an area of resolution biology that has been studied in much detail and, as a consequence, has the potential to provide numerous targets for pro-resolution drug development. Here, we discuss the many 
fates of cells during resolution, with a focus on the local death of PMNs and their efferocytosis by mononuclear cells.

Leukocyte death. Inflammatory cells can be eliminated in one of three ways: through retro-transendothelial migration back into systemic circulation; through lymphatic drainage (to enable subsequent participation in adaptive immune responses); or through cell death in the inflamed tissue. Local cell death occurs in many ways, including autophagy, excitotoxicity, pyroptosis, necrosis, necroptosis, NET-osis and caspase-mediated apoptosis 69,70 (Box 2). The predominant mechanism of cell death in a given disease state is not without reason or consequence: instead it plays an active part in determining the outcome of inflammation.

Granulocyte death by apoptosis during resolution has been extensively studied (see below). Key molecules and pathways involved in maintenance of leukocyte survival or apoptotic death include NF-KB, phosphoinositide 3-kinase (PI3K), myeloid leukemia cell differentiation protein 1 (MCL1) and CAMP. Regulated clearance of apoptotic cells leads to shutdown of immune cellular activity (in particular efferocytosing macrophages) and the consequent inhibition of inflammatory responses. Therefore, it is considered that granulocyte apoptosis followed by phagocytosis (efferocytosis) is the most 'desirable' form of cell death for successful resolution ${ }^{71}$.

Granulocytes survive in the circulation for only a few hours, but various soluble mediators released at sites of inflammation can influence the survival of diverse granulocyte subtypes. For example, PMN longevity is extended by IL-6, IL-8, GMCSF and bacterial products, whereas eosinophil survival is augmented by cytokines such as IL-3, IL-5 and GM-CSF ${ }^{72}$. These survival factors, in turn, activate several signalling pathways including the cyclin-dependent-kinase (CDK), NF-kB, PI3K-Akt, cAMP and mitogen-activated protein kinase (MAPK) pathways ${ }^{73}$. Generally, these signalling pathways lead to enhanced levels of pro-survival molecules and diminished levels of pro-apoptotic molecules. NF-kB, in particular, alters the expression of many intracellular pro-survival proteins, including BCL-2 family members (such as BCL-xL and XIAP). Therefore, pharmacological inhibition of NF$\mathrm{KB}$ and IKKa influences granulocyte survival and apoptosis and consequently drives inflammatory resolution ${ }^{74}$.

In addition to NF-KB, the intracellular pro-apoptotic protein BAD (BCL-2-associated death promoter) is an important downstream target of PI3K-AKT. AKT phosphorylates BAD, thereby suppressing apoptosis and promoting cell survival ${ }^{75}$. In myelin oligodendrocyte glycoprotein-induced experimental autoimmune encephalomyelitis (a model of multiple sclerosis), PI3Ky-deficient mice developed milder clinical disease and decreased levels of CCL2 and CCL5 in brain tissue compared with wild-type controls, concomitant with increased leukocyte apoptosis in the PI3Ky-deficient animals ${ }^{76}$. Importantly, neither PI3Ky-deficiency nor the PI3K inhibitor AS-605240 affected the magnitude of the acute onset phase of the disease, indicating that in this model, $\mathrm{PI} 3 \mathrm{~K} y$ may have a role in promoting leukocyte survival - preventing resolution and extending inflammation. 
The role of cAMP in apoptosis is complex as in some cells types is drives programmed cells death, while others it prevents it ${ }^{77}$. For instance, in vitro cAMP inhibits granulocyte apoptosis and the subsequent recognition and uptake of apoptotic cells by macrophages ${ }^{78,79}$. However, increasing cAMP function in vivo in LPS-induced pleurisy (with the phosphodiesterase 4 inhibitor rolipram, the adenylate cyclase activator forskolin or the cAMP analogue db-cAMP) 4 hours after LPS challenge dose-dependently prevented PMN accumulation but, in contrast to in vitro studies, promoted PMN apoptosis ${ }^{80}$. Independent of its effects on phagocytosis of apoptotic cells, rolipram causes a direct, cAMP-dependent switch of M1-like proinflammatory macrophages to resemble those found during resolving inflammation ${ }^{81}$. Collectively, these findings show that cAMP signalling regulates processes that limit tissue injury, and that modulation of cAMP levels represents a central therapeutic strategy in the control of resolution. However, this will most likely be context, tissue and stage of inflammation dependent.

All MAPK subtypes (namely, extracellular-signal-regulated kinase 1 (ERK1) and ERK2, c-Jun amino-terminal kinases (JNKs) and p38 MAPK) play a part in modulating granulocyte apoptosis. However, their respective roles in cell survival are cell-type- and context-specific ${ }^{82}$. ERK is typically associated with the regulation of activation and survival of various cell types, including granulocytes ${ }^{83}$. In carrageenan-induced pleurisy, for instance, the ERK1 and ERK2 inhibitor PD98059 hastens resolution of inflammation, with fewer PMNs and macrophages in the pleural cavity and increased PMN apoptosis ${ }^{84}$. By contrast, the exact role of p38 in the control of granulocytes survival is controversial; this pathways has been described to have both pro-apoptotic ${ }^{85}$ and anti-apoptotic ${ }^{86}$ effects. During resolution, the restriction of p38 MAPK activation by MAPK phosphatase 1 controls the pro- to antiinflammatory macrophage polarization switch during muscle healing ${ }^{87}$. Further, during the non-phlogistic phagocytosis of apoptotic cells, the p38 signalling pathway leads to the activation of certain transcription factors that reprogramme the macrophage phenotype from pro- to anti-inflammatory, resulting in release of IL-1088.

A role for CDK in granulocyte survival and in delaying resolution in experimental animal models was illustrated with the use of the CDK inhibitor roscovitine, which inhibits the ability of CDK7 and CDK9 to phosphorylate RNA polymerase $1{ }^{89}$. This compound caused apoptosis of GM-CSF-, LPS- or glucocorticoid-stimulated human PMNs $^{90,91}$. Additionally, roscovitine and AT7519 (a CDK inhibitor in clinical trials for treatment of cancers, neurodegenerative diseases, viral infections and glomerulonephritis) dose-dependently induced apoptosis of human eosinophils ${ }^{92}$. Importantly, in vivo, roscovitine enhanced the resolution of established PMNdependent inflammation in models such as carrageenan-induced pleurisy, carrageenan-induced arthritis and bleomycin-induced lung injury ${ }^{90}$. Moreover, in a murine model of ovalbumin-induced allergic pleurisy, AT7519 triggered eosinophil apoptosis and subsequent phagocytic clearance ${ }^{92}$. These data highlight the importance of phagocytic clearance of inflammatory cells to the resolution process.

Collectively, targeting death pathways of pathogenic cells that persist at sites of chronic inflammation has its virtues. The effectiveness of this approach will depend on being able to selectively target cells of interest, such as PMNs, eosinophils or Th1-type lymphocytes, at the right site, while leaving pro-resolving, pro-homeostatic and bystander cells intact. 
Clearance of dead cells. Defective clearance of apoptotic bodies has been linked with autoimmunity ${ }^{93}$ and other chronic inflammatory diseases ${ }^{94}$; as a result, efferocytosis has been considered a key requirement for inflammatory resolution and for the preservation of immune tolerance ${ }^{71}$. When a PMN nears the end of its life it releases chemoattractants, including nucleotides ${ }^{95}$, lysophosphatidylcholine ${ }^{96}$ and sphingosine-1-phosphate ${ }^{97}$, that signal its whereabouts to mononuclear phagocytes. In addition, endothelial monocyte-activating polypeptide II98, ribosomal protein S1999 and thrombospondin $1^{100}$ direct mononuclear phagocytes towards apoptotic cells. Interestingly, it has been suggested that apoptotic cells also release resolvin E1 (RvE1) and protectin D1 101 , lactoferrin and annexin A1, which inhibit further recruitment of granulocytes but not of mononuclear phagocytes. This selectivity may contribute towards the predominance of mononuclear cells that mediate resolution and optimal wound healing ${ }^{102}$.

During apoptosis, the dying cell loses expression of repellant 'don't-eat me' cellsurface molecules such as CD31 ${ }^{103}$ and CD47 ${ }^{104}$, and upregulates expression of various 'eat-me' signals, including phospholipids, nucleotides and phosphatidylserine (PS), which undergo externalization ${ }^{105}$. The interaction between tissue-resident macrophages and these 'eat-me' signals ${ }^{105-109}$ ensures that apoptotic cells are efficiently removed from tissue prior to membrane rupture and the release of histotoxic mediators that would theoretically damage and perpetuate the inflammatory response.

Importantly, uptake of apoptotic cells trigger the conversion of phagocytosing macrophages to an immune-regulatory or pro-resolution phenotype ${ }^{81,110}$, limiting their propensity for tissue damage and maintaining immune tolerance both locally and in draining lymphoid organs ${ }^{4,111-113}$. This phenotype is characterized by upregulated expression of co-inhibitory molecules such as PDL1 and ICOS ligand; the release of the anti-inflammatory cytokines IL-10 and TGF $\beta$; the secretion of PCNA-associated factor, $\mathrm{PGE}_{2}$ and CAMP81, 114-116; and the inhibition of the release of pro-inflammatory cytokines including TNF, GM-CSF, IL-12, IL-1 $\beta$ and IL-1825, 59-61. Macrophages that have ingested apoptotic cells also release pro-resolving lipid mediators such as RvE1, protectin D1 and maresins ${ }^{101}{ }^{117}$, contributing to termination of the inflammatory process.

There are hypothetical benefits in therapeutically triggering apoptosis accelerate resolution, but this must be accompanied by robust efferocytosis, as apoptotic cells and their associated auto-antigens potentially underlie disease progression in SLE ${ }^{93}$. Moreover, as discussed below, efferocytosis is an immunosuppressive process and so opens a window of potential infectious opportunity ${ }^{118}$. Therefore, a better understanding of the interaction between apoptotic cells and phagocytosis, and the soluble mediators that modulate this process will be important in understanding both the pathogenesis of chronic diseases and delineating novel ways of treating them.

\section{Macrophage phenotypes during resolution}

Early studies on acute inflammation suggested that PMN clearance and the restoration of tissue homeostasis represented the end of successful resolution ${ }^{2,17 .}$ However, as described above, we recently reported on a third phase of inflammation, 
which we called 'post-resolution', in a murine model of zymosan-induced peritonitis, suggesting that the classic view of resolution needed to be expanded ${ }^{4}$.

This phase persisted for weeks and involved populations of Ly6chi MDSCs, DCs and macrophages. These monocyte-derived cells amplified the ensuing adaptive immune response and maintained immune tolerance by generating inducible regulatory $T$ cells ${ }^{4}$. We $\mathrm{W}^{4}$ and others ${ }^{10}$ also found that Ly $6 \mathrm{c}^{\text {hi }}$ MDMs remained in tissues for months after inflammation had resolved, and that these cells had a role in dictating the magnitude and duration of subsequent acute innate inflammatory stimulation (remniscent of studies of polysensitization). In another study in mice, DCs were found not to return to their steady-state phenotype after resolution of Th2-mediated inflammation, but instead persistently expressed high levels of co-stimulatory molecules, such as CD80, CD86, and intercellular adhesion molecule 1 (ICAM1), and the Th2-skewing molecules OX40 ligand and Ym1/2 lectin. This enhanced maturation status of airway DCs facilitated neosensitization up to at least 1 month after inflammation ${ }^{119}$. These findings emphasize that once inflammation has resolved, there is a hitherto unappreciated phase of 'adapted homeostasis', in which cells of the monocyte-macrophage lineage play a central part in modulating subsequent responses to infection or allergens.

Therefore, despite experiencing the same inflammatory cues, monocyte or macrophage populations might come to possess diverse phenotypes that are neither M1 (pro-inflammatory) nor M2 (anti-inflammatory), but commensurate with the phase of inflammation. Indeed, this notion probably extends to macrophage populations in tissue niches under different physiological and disease conditions ${ }^{120,}{ }^{121}$. For instance, during the maximal fibrotic stage of liver fibrosis elicited by carbon tetrachloride, Ly6 $\mathrm{c}^{\text {hi }}$ monocytes give rise to $\mathrm{MDMs}^{122}$. These macrophages are a hybrid of the conventional M1- and M2-like cell phenotypes and express elevated levels of growth factors and tissue remodelling-related factors needed to clear fibrotic plaques, and exhibit elevated ERK signalling. Interestingly, using liposomes to elevate ERK signalling enhanced anti-fibrotic macrophage phagocytosis in vivo and found that pharmacologically elevating the phagocytic nature of their anti-fibrotic macrophage using liposomes accelerated the resolution of liver fibrosis.

Data from these reports support the idea of macrophage plasticity - that the macrophage phenotypes that either drive or resolve diseases will be tissue-, stimulus- and phase-specific. Indeed, diverse populations of macrophage phenotypes have been reported in various experimental models of renal (that is, single-organ) inflammation (for review see ${ }^{123}$ ). The precise nature of macrophage phenotypes involved in each disease will depend on which disease mechanism predominates in a given organ. Whereas infection and cell necrosis trigger the generation of pro-inflammatory or $\mathrm{M} 1$-like macrophages that exacerbate renal cell damage, apoptotic cells induce anti-inflammatory, immune-suppressive macrophages that promote epithelial and vascular repair. Indeed, vascular and epithelial healing that is insufficient despite abundant growth factor secretion will promote profibrotic 'M2a' or 'wound-healing' macrophages that accelerate fibrogenesis. In a separate example, inflammatory monocytes recruited to sites of skeletal muscle injury switched into anti-inflammatory macrophages to support myogenesis $^{124}$ in a manner dependent on $\mathrm{AMPKa} 1^{125}$, a master regulator of energy 
homeostasis. Thus, like the peritoneum ${ }^{4}$, a single organ like the kidney, or tissue such as skeletal muscle, can generate diverse populations of immune cells depending on disease aetiology.

These data indicate that there are many diverse routes to resolution; thus, it is unlikely that one single therapeutic intervention will be a panacea for all chronic inflammatory diseases. Instead, it will be increasingly imperative to focus on a specific pathology, appreciating both the heterogeneity within classical clinical diagnoses and the factors that influence the inflammatory phenotype, to successfully admin targeted pro-resolution therapy is to be.

\section{Targeting pro-resolution pathways}

The aspects of the pro-resolution cascade described above can be targeted in order to alter the inflammatory profile, without compromising tolerance. Modulating any of these events, along with supplementation of, or inhibition of the catabolism of, soluble pro-resolution factors to extend their pharmacological half-life, represent tractable drug targets to invoke resolution. Table 1 describes most of the currently known mediators, receptors and mechanisms that exert pro-resolution actions.

The number, intricacy and applications of human inflammatory models are increasing (Box 3), but zebrafish and mice (Box 4) have provided us with the quantifiable resolution indices we use in these models. These indices include the maximum cell number present at the peak of the inflammatory response $\left(\Psi_{\text {MAX }}\right)$, the time at which $\Psi_{\text {MAX Occurs }}$ (TMAX), and the time at which cell numbers are reduced by $50 \%$ of $\Psi_{\text {MAX }}\left(T_{50}\right)$. The resolution interval $\left(R_{i}\right)$ is the time taken for cells at $\Psi_{\text {MAX }}$ to reach $T_{50}$ (that is, to be reduced by $\left.50 \%\right)^{126}$. These metrics allow comparative efficacy study of existing and novel pro-resolution therapies.

In terms of modulating cell recruitment to sites of infection or injury, and as mentioned above, it is important to differentiate between (anti-inflammatory) factors that only inhibit granulocyte trafficking into tissues (such as metformin ${ }^{127}$ ) versus those that that are also pro-resolution. For instance, lipoxins and resolvins (Rvs) inhibit PMN trafficking and enhance efferocytosis of apoptotic cells, without compromising host defence ${ }^{128}$, and RvD1, RvD5 and protectin D1 accelerate leukocyte bacterial killing and enhance antibiotic efficacy ${ }^{25}$.

There are several strategies for enhancing PMN apoptosis in vivo - including CDK inhibition (using roscovitine or AT7519), or inhibition of PI3K, ERK1, ERK2, or NF-KB (using the $\mathrm{PGD}_{2}$ metabolite 15-deoxy $\left.\Delta^{12-14}-\mathrm{PGJ}_{2}\right)^{129}$. However, for maximum benefit, this must be accompanied by efferocytosis by macrophages. This can be augmented by melanocortins, glucocorticoids, annexin A1 and its mimetic Ac2-26 as well as lipid mediators ${ }^{130}$. Recently, Bc/2-overexpressing mice showed impaired PMN apoptosis in a model of pneumococcal meningitis, and this effect was accompanied by high levels of IL-1 $\beta$ and granulocyte-CSF, and reduced levels of anti-inflammatory TGF $\beta$, resulting in persistence and prolonged activity of PMNs and thus more-severe disease ${ }^{131}$. Inducing PMN apoptosis using the CDK inhibitor roscovitine improved thereduced brain tissue damage and accelerates recovery following pneumococcal meningitis. 
Although hastened PMN apoptosis and the resultant re-programming of macrophages towards an immune-modulatory or pro-resolution phenotype may be beneficial, resolving inflammation before the tissue is fully immunologically restored may open a window of 'infectious opportunity'. The lung, for instance, is highly susceptible to secondary bacterial infections as a consequence of injury. Apoptotic cells suppress in vitro phagocytosis and killing of bacteria by alveolar macrophages in a PGE $2^{-}$, prostaglandin EP2 receptor (PTGER2)- and cAMP-mediated manner ${ }^{118}$. Intrapulmonary administration of apoptotic thymocytes impaired lung recruitment of PMNs as well as clearance of Streptococcus pneumonia, again through a PGE2- and PTGER2-dependent pathway. These results suggest that, in addition to their beneficial homeostatic influence, pro-resolution programmes, at least in the lung, may dampen innate antimicrobial responses, leaving the host susceptible to secondary infection and perhaps necessitate the concomitant use of immunerestorative agents such as PTGER2 antagonists to counteract these effects ${ }^{34}$. Moreover, the contrast between these findings from different infected sites (meninges and lung) re-emphasizes that the impact of various pro-resolution pathways on tissue integrity, repair and susceptibility to infection are most likely disease- and tissue-specific.

Lipid mediators such as $\mathrm{PGJ}_{2}$ and RvD1 exert multiple levels of control on the inflammatory response, and mimetics based on their mode of action through $\mathrm{g}$ protein-coupled receptors (GPCRs) represent a tractable and very effective target for treating ongoing inflammation ${ }^{132}$. $\mathrm{PGJ}_{2}$ was one of the first COX-derived lipid mediators implicated as eliciting the resolution of innate-immune ${ }^{133}$ as well as regulating adaptive-immune inflammatory responses ${ }^{134-136}$. It is an agonist of the GPCR PPARy, and represses the transcription of mRNAs encoding proinflammatory mediators, inhibits protein translation ${ }^{137}$ and inhibits NF-kB-mediated transcription of pro-inflammatory genes ${ }^{138}$. $\mathrm{PGJ}_{2}$-mediated translational repression triggers a stress response, resulting in the assembly of stress granules ${ }^{139,} 140$ that reprogramme gene expression to allow cells to survive noxious stimuli. Complimentarily, RvD1 upregulates miR-146 to control NF-kB expression ${ }^{141}$. RvD1 also upregulates miR-219 and miR-208a (the latter of which triggers IL-10 expression) in exudate cells isolated from the GPCR $N$-formyl peptide receptor 2 (FPR2; also known as ALX)-overexpressing transgenic mice bearing a zymosaninduced peritonitis. In Fpr2 knockouts, RvD1 had no effect on leukocyte infiltration, did not regulate miR-208a, and had no subsequent effect on IL-10 expression ${ }^{142}$, suggesting the actions of RvD1 are dependent on FPR2. Targeted, stratified application of either of these agents, either locally or systemically, could thus potentially help drive resolution in inflamed tissues.

It must be emphasised that the targets discussed above are involved in pathways pertinent to acute, self-resolving inflammation. Whereas it is relatively easy to make already-resolving experimental inflammation resolve even faster, it is far more challenging, though more clinically relevant, to achieve this in diseases of different aetiology, chronicity and complexity. Granulomatous experimental autoimmune thyroiditis (G-EAT) is a T cell-mediated autoimmune disease that can be induced in genetically susceptible strains of mice by immunization with mouse thyroglobulin and adjuvant ${ }^{143}$. It is characterized by proliferation of thyroid epithelial cells, granuloma formation and infiltration of $\mathrm{T}$ lymphocytes, macrophages, multinucleated giant cells and PMNs. Neutralizing the endogenous pro-apoptotic molecule TNF-related 
apoptosis-inducing ligand (TRAIL) in this model has little effect on the development of the lesion, but markedly inhibited lesion resolution ${ }^{144,}{ }^{145}$. In mice that received a TRAIL-specific antibody, the inhibition of resolution correlated with the expression of TRAIL and of the anti-apoptotic molecules FLICE-like inhibitory protein (FLIP) and $B C L-X L$ on thyroid inflammatory cells. These results suggest that endogenous TRAIL is not required for G-EAT initiation and development but is crucial for G-EAT resolution. Furthermore, TRAIL accelerated PMN apoptosis and resolution in LPSmediated acute lung injury and in zymosan-induced peritonitis ${ }^{146}$. Therefore, TRAIL may promote resolution of chronic inflammatory disease process, at least in part through altering the expression pattern of pro- and anti-apoptotic molecules of both tissue stromal and inflammatory cells. The example of TRAIL emphasizes that the resolution of complex chronic diseases (as well as of acute inflammation) is therapeutically achievable.

Whereas the targets discussed here and elsewhere were empirically found to promote resolution, there are other drugs that exert striking anti-inflammatory as well as pro-resolution effects. Specifically, $\mathrm{H}_{2} \mathrm{~S}$ has the anti-inflammatory effects of inhibiting granulocyte adhesion to, and transmigration across, the microvascular endothelium, and inhibiting myeloperoxidase activity, but also helps resolve inflammation by promoting the apoptosis of PMNs, the uptake of bacteria by phagocytes and the acquisition of an M2-like phenotype by macrophages ${ }^{147}$, while promoting tissue repair and restoration of tissue function ${ }^{148}$. Some of the proresolution properties of $\mathrm{H}_{2} \mathrm{~S}$ arise from its ability to selectively induce expression and activity of inducible COX, which produces pro-resolution lipid mediators. Inhibition of $\mathrm{H}_{2} \mathrm{~S}$ synthesis in the gut, for example, leads to reduced COX expression, an increase in mucosal inflammation and impaired healing of experimentally damaged tissue - effects that may be rescued by $\mathrm{H}_{2} \mathrm{~S}$ donors. As a consequence, several groups began to try to develop novel therapeutics that release $\mathrm{H}_{2} \mathrm{~S}^{149}$. ATB-429 from Antibe Therapeutics is a derivative of mesalamine that releases $\mathrm{H}_{2} \mathrm{~S}$. ATB-249 exhibits markedly enhanced anti-inflammatory, pro-resolution and pro-healing effects in rodent models of colitis compared with mesalamine, whereas the $\mathrm{H}_{2} \mathrm{~S}$-releasing NSAID ATB-346 (which is in Phase I for osteoarthritis), accelerates gastrointestinal wound repair, in stark contrast to its parental compound, naproxen, which causes gastrointestinal lesions ${ }^{149}$. Such agents clearly demonstrate the potential of the incipient class of pro-resolving therapies.

\section{Future strategies to target resolution}

Many advances have been made in understanding resolution and its constituent processes; now, we must learn how to control them with convincing efficacy. For example, a range of factors have a role in inflammatory resolution, including lipids (such as lipoxins, Rvs, protectins, maresins and PGD2 or cyclopentenone PGs), proteins (such as annexin A1 and secretory leukocyte protease inhibitor), proteins (such as annexin-, melanocortin- and chemerin-derived peptides), gaseous mediators (such as $\mathrm{H}_{2} \mathrm{~S}$ and carbon monoxide (CO)), purines (such as adenosine) as well as the vagal nerve release of acetylcholine into the affected tissue. The question of where, when and how to therapeutically employ or manipulate these moieties remains. 
Existing anti-inflammatories also possess pro-resolution properties: low-dose aspirin, triggers the production of epimeric forms of lipoxins (15 epi-lipoxin $A_{4} / B_{4}$ ), and glucocorticoids enhance efferocytosis. However, such drugs have unwanted side effects. It now clear though that a better understanding of their pharmacological mechanisms of pro-resolving action may be able to obviate these. For example, recent insights into melanocortin receptors have reinvigorated interest in the use of adrenocorticotophic hormone $(\mathrm{ACTH})$ as a drug. ACTH not only induces cortisol production, but also exerts anti-inflammatory actions by: targeting melanocortin receptors present on immune cells; inhibiting granulocyte trafficking; suppressing cytokine synthesis; and driving macrophages towards an M2-like phenotype. These findings suggest that new ACTH-like melanocortin receptor-targeting drugs devoid of steroidogenic actions could possess potent pro-resolution effects ${ }^{150}$. Equally, forms of lipoxins that are endogenously produced following exposure to aspirin (known as aspirin-triggered lipoxins (ATLs) or long-lived mimetics of these may afford similar benefits to aspirin without the gastrointestinal and haemorrhagic sequelae.

Theoretically, pro-resolution therapies may be superior to standard antiinflammatories, in that as well as suppressing the first four cardinal signs of inflammation, they may also affect tissue healing and function. Early evidence comes from murine studies of inflammation-induced bone loss. Pro-inflammatory cytokines such as TNF and IL-1 promote pathologic osteoclast resorption of bone and suppress the ability of osteoblasts to counteract bone erosion; however, during the resolution phase of the mouse $\mathrm{K} / \mathrm{BxN}$ serum-induced inflammatory arthritis model, bone resorption ceased and appositional osteoblast-mediated bone formation was induced $^{151}$. Remarkably, this resulted in repair of eroded bone - probably as a result of downregulation of the Wnt antagonists secreted frizzled-related protein 1 (sFRP1) and sFRP2, and concomitant induction of the anabolic and pro-matrix mineralization factors Wnt10.

Finally, and potentially most importantly, the translation of pro-resolution strategies to man is underway. The findings that membrane anti-inflammatory GPCRs including ChemR23, GPR32 and FPR2 transduce the pro-resolving effects of chemerin peptides, RvE1 and RvD1 (and that RvD2 exerts its effects through GPR18 ${ }^{152}$ ) are not only novel and exciting developments in receptor biology, but may represent an important opportunity for pro-resolution drug discovery. A better understanding of these receptors, especially in chronic inflammatory settings, could guide novel drug discovery programmes aimed at using the fundamental actions of these effectors of resolution. This process has already started with a stable isopropyl ester analogue of RvE1, RX-10045 (Resolvyx Pharmaceuticals), which is being tested for its ability to resolve dry eye inflammation (Clinicaltrials.gov identifier: NCT00799552).

\section{Conclusion and future directions}

The development and application of pro-resolution therapeutic strategies to chronic inflammatory pathology may come to revolutionize the management of some of the oldest and most prevalent human ailments. By approaching such diverse disease states from the opposite perspective - asking not how to suppress the initiation and propagation of inflammation, but how to augment clearance of the triggering stimuli and resolve the host response to it - we will hopefully gain new insights into both the origin of chronic inflammation and pharmaceutical means of addressing it. 
This article has described compelling evidence that modification of the pathways that initiate pro-resolution processes (removal of the stimuli, dampening of proinflammatory signalling and enhanced mediator catabolism) and these key events themselves - apoptosis of infiltrating inflammatory leukocytes, the clearance of these cells, and associated alterations in macrophage phenotype - can improve the outcome of disease in animal models. Although the medicinal means of directing and orchestrating these fundamental processes are currently in their infancy, the potential reward from harnessing pro-resolution pathways is too great to ignore.

Various promising strategies have been elucidated; nevertheless, there is a pressing need to develop appropriate human models of resolution in which their efficacy can be validated. Given the heterogeneity of inflammatory disease and the challenges posed by co-morbidity, polypharmacy and demographic variance in the clinical population, it is implausible that one 'pro-resolution drug' will be a panacea. Instead, the thoughtful development of a stratified approach in targeted patient groups, accounting for tissue-site differences in resolution pathways and disease-specific factors, is most likely to bear fruit.

\section{Box 1 | The role of PMNs in resolution and adaptive immunity}

Notwithstanding the undoubted function of PMNs in killing infection, there is a presupposition that PMNs are 'bad' in self-resolving inflammation - the context in which pro-apoptotic or pro-efferocytosis drugs have been tested. Previously, we made a link between the classic model of acute inflammatory resolution and the establishment of adaptive immunity. Others have also made important observations that indicate a hitherto unappreciated role for PMNs in this process, including that of 'lymph node cell shutdown'.

The term lymph node cell shutdown describes how - unlike in the steady state, when the rate of lymph flow and the output of cells in the efferent lymph is constant - there is a transitory decrease of lymphocytes exiting the efferent lymph within hours of antigen or adjuvant injection ${ }^{153-155}$. Lymph node shutdown is thought to facilitate the development of adaptive immunity. Lipid mediators including $\mathrm{PGE}_{2}$ and thronboxane $A_{2}$ are thought to be the critical component in this process ${ }^{154,156 .}$

A later study showed that within minutes of infection or vaccination, PMNs transit to regional draining lymph nodes and suppress ensuing CD4 and B cell responses in a thromboxane-mediated process ${ }^{157}$. While its belived that PMN prostanoids directly inhibit $\mathrm{T}$ cell and $\mathrm{B}$ cell responses by inhibiting their proliferation; recent studies suggest that PGE2, for instance drives CD4 Th1 immune cells resposnes ${ }^{158}$. In addition, PMNs compete with DCs for antigen, limiting antigen availability to DCs; such competition may further compromise the quality of adaptive immune responses.

Thus, while we appreciate the established role PMNs have as first-line defenders against pathogens, these cells also play an important part in modulating adaptive immune responses. Whether these effects are beneficial to the host depends on the nature of the inflammatory stimulus - infection or vaccination. However, what is clear is the need to understand the broader role PMNs have during acute inflammation, and the impact that hastening PMN apoptosis may have on the nature and magnitude of the ensuing adaptive immune response. Moreover, the role of 
PMNs in modulating $\mathrm{T}$ cell or $\mathrm{B}$ cell responses in chronic inflammation that is driven by maladaptive immunity warrants further investigation. It is for this reason that we need to develop more-reflective models of chronic inflammation, to establish the overall virtues and limitations associated with hastening PMN apoptosis, as the role of granulocytes in chronic, maladaptive immunity may not be the same as in acute inflammation.

\section{Box 2 | Alternative forms of cell death in inflammation}

Besides apoptosis, there are several alternative forms of cell death that can occur following inflammation, including autophagy, neutrophil extracellular trap (NET)-osis and necrosis.

Autophagy is the ordered degradation and recycling of unnecessary or dysfunctional intracellular components. Autophagic cell death occurs after nutrient deprivation or growth factor withdrawal or in response to rapamycin or inflammatory-like stimuli such as TLR agonists, phorbol myristate acetate (PMA) or reactive oxygen species. It typically occurs without chromatin condensation and is accompanied by cytoplasm vacuolization ${ }^{159}$. Autophagy provides a mechanism for the elimination of intracellular microorganisms. It also controls inflammation - through regulatory interactions with innate immune signalling pathways, by removing endogenous inflammasome agonists and through effects on the secretion of immune mediators ${ }^{160}$.

In addition to autophagy, PMN extracellular traps, or NETs, that are composed of DNA and granule constituents lead to non-apoptotic cell death termed NETosis ${ }^{161}$. Although NETs are designed to kill bacteria and fungi, they are proposed to worsen inflammation. Consistent with this, PMNs isolated from synovial fluid or peripheral blood of patients with acute gout - an inflammatory condition associated with the accumulation of uric acid in synovial joints - produce NETs ${ }^{162}$.

Cells may also undergo necrosis in response to signalling via TNF family death receptors, pathogen sensors, antigen-specific $T$ cell receptors and genotoxic stress. Necrosis can occur either as a primary event (in which case, it is known as primary necrosis) or secondary to apoptosis, if apoptotic bodies are not immediately cleared (as in the presence of pathogen-encoded caspase inhibitors) ${ }^{163}$. Necrosis was traditionally viewed as an uncontrolled mode of cell death until it was found that certain types of necrosis are tightly regulated by signal transduction pathway. In particular, the protein serine-threonine kinase receptor interacting protein 1 (RIP1) family member RIP3 is now considered a key mediator in caspase-independent cell death ${ }^{163,}{ }^{164}$, which is now known as necroptosis or programmed necrosis. Some pathogens in monocyte-macrophages, PMNs and eosinophils can disrupt death pathways as a survival strategy, impairing antimicrobial functions of immune cells ${ }^{165}$, 166.

Although necrosis has been traditionally associated with enhanced inflammation and tissue injury and therefore considered to be 'unwanted' 167 , it has been suggested that necrotic cell death can inhibit inflammatory reactions ${ }^{168}$. For example, annexin A1, a glucocorticoid-inducible anti-inflammatory and pro-efferocytotic protein, is externalized on granulocytes during secondary necrosis. Here, it acts as an immune- 
dampening system, counteracting inflammatory responses when apoptotic cells are not cleared ${ }^{169}$.

The relative virtues of necrotic cell death on inflammation are beyond the scope of this Review, but these findings muddy the waters in terms of what was once thought to be good cell death (apoptosis) versus bad death (necrosis). Indeed, while autophagic-like PMNs have been reported in rheumatoid arthritis, septic shock and cystic fibrosis ${ }^{170,171}$, a recent study on the inflammatory joint disease gout proposed that aggregated NETs promote the resolution of PMN-driven inflammation, by degrading cytokines and chemokines and disrupting PMN recruitment and activation ${ }^{172}$. Thus, as the mode of cell death during resolution may be dependent on tissue environmental cues, it may also be tissue- and disease-specific.

\section{Box 3 | Challenges with translation into humans}

Animal models of inflammation and resolution (Box 4) have generated invaluable information on cell trafficking, cell clearance as well as the receptors and soluble mediators that control these processes; howvever, their relevance to human physiology and pathology has been questioned. The disparity between rodent and human experimental medicine was exemplified by a recent report showing that although acute inflammatory stimuli such as trauma, burns and endotoxin result in highly stereotyped genomic responses in humans, the equivalent responses in mouse models were not comparable ${ }^{173}$. Although controversial ${ }^{174,}{ }^{175}$, Seok et al. called for "higher priority for translational medical research to focus on the more complex human conditions rather than relying on mouse models to study human inflammatory diseases"173. This is not without difficulty. Studying fundamental resolution biology in the clinical population is challenging, being complicated by demographic variables, polypharmacy, sample timing with regards to disease stage and other factors. Further, there are inevitably complex interactions between concomitant pathologies and resolution pathways, as exemplified by atherosclerosis and hyperglycaemia.

Atherosclerosis is initiated by shear stress and the deposition of cholesterol-rich lipoproteins in the artery wall with the resultant entry of inflammatory leukocytes into lesions. This process, if left un-checked, impedes resolution, and enables disease progress to endothelial dysfunction, vascular smooth muscle proliferation and associated clinical sequelae ${ }^{176}$. Reducing plasma low-density lipoprotein cholesterol levels using rosuvastatin or atorvastatin can promote regression of atherosclerotic lesions in humans ${ }^{177}$ - in other words, removing the inciting stimulus is sufficient to help resolve this multifactorial chronic inflammatory disease. However, this statinmediated effect is either obscured or negated in individuals with type 1 or type 2 diabetes. In murine models, hyperglycaemia is associated with increased numbers of circulating monocytes - especially inflammatory Ly6C $^{+}$monocytes ${ }^{178}$ - and PMNs ${ }^{179}$, which leads to their increased, proportional entry into plaques and consequent accelerated lesion progression. This appears to be replicated clinically: compared with individuals who have atherosclerosis alone, individuals with atherosclerosis and diabetes show impaired regression in response to cholesterolreducing therapies ${ }^{180}$, emphasizing the challenge of attempting to pharmacologically resolve clinical disease. 
Perhaps the optimal pragmatic course for demonstrating the potential of proresolution strategies would be a combination of studies Blume, K.E. et al. Cell surface externalization of annexin A1 as a failsafe mechanism preventing inflammatory responses during secondary necrosis. J Immunol 183, 8138-47 (2009). on healthy humans and rodents. To this end, several human models to explore innate immunity and inflammation are now available. Skin represents a reproducible and clinically-relevant window into the human immune system, with multiple methods to elicit inflammation - including cantharidin ${ }^{181-184}$, which causes acantholysis. This sterile tissue injury model is driven by damage-associated molecular pattern molecules (DAMPs) such as purines, S100 proteins and nucleic acids. The elicited inflammatory response is characterized by rapid PMN infiltration followed by recruitment of macrophages and a smaller proportion of lymphocytes. Although responsive to many traditional antiinflammatories, the model's utility is limited in temporal duration $(<72 \mathrm{hrs})$ by blister stability. Other models include intradermal injection of endotoxin ${ }^{185}$ or lung inhalation of endotoxin ${ }^{186}$. Both trigger a robust innate immune response that spontaneously resolves and may better faciliitate exploration of post-resolution processes, offering a more 'physioloigical' system. Akbar and colleagues have additionally characterized the adaptive immune responses to recall antigens, including tuberculin purified protein that was administered via intradermal injection and subsequent suction blister, as a means of understanding immunosenescence in aged human volunteers ${ }^{187,188}$.

Demonstration of the similarities of the fundamental biological processes that underlie resolution in these human models with those observed in animal studies to date will hopefully provide reassurance of their validity. Further development and appropriate selection of human inflammatory models, along with acquisition of dedicated clinical samples, will, however, be crucial in translating current theory to future therapy.

\section{Box 4 | Murine and zebrafish models}

In mice, the effects of putative and anti-inflammatory and pro-resolution drugs can be traced over time using the 'industrial standard' oxazalone ear-swelling (type IV hypersensitivity) and carrageenan-induced paw-swelling models. These models also enable each animal to serve as its own control, meaning smaller numbers of mice need be used - particularly useful when using precious transgenic mice or in quick proof-of-principle investigations. Whereas these models' readouts centre on oedema formation, they may be complemented with peritonitis and pleuritis models that use sterile stimuli such as zymosan, which allow greater insight into leukocyte dynamics and access to inflammatory exudates that contain soluble mediators.

It is important to bear in mind idiosyncratic features of individual models of different diseases in different species, genetic manipulations, and organs or tissues. For example, it is ill-advised to use transgenic mice with the air-pouch model, owing to the possibility that the intra-pouch fibro-vascular lining that is needed to enumerate the inflammatory response may be dependent on expression of the gene of interest during development, although this concern can be mitigated by using conditional knockouts.

Originally, termination of PMN influx, efferocytosis and cytokine clearance were considered adequate metrics of resolution; however, these quickly became 
insufficient ${ }^{17,}{ }^{189}$. Quantitative indices such as $\Psi_{\max }$ (the maximal number of PMNs present during the inflammatory response), $T_{\max }$ (the time at which $\Psi_{\max }$ occurs) and the resolution interval $\left(R_{i}\right)$ from $T_{\max }$ to $T_{50}$ (when PMN numbers reach half $\left.\Psi_{\max }\right)^{126}$ became useful when quantifying the effects of pharmacological intervention or genetic manipulation on resolution. In turn, a new, more-robust set of criteria will probably be required to assess the changes in the ability of resolution processes to bridge the gap between acute inflammation and adaptive immunity.

Zebrafish can also be used in inexpensive high-throughput screening of proresolution drugs. For example, tail fins can be surgically transected to cause a reproducible and robust inflammatory response that can be assessed by PMN counts or through fluorescence imaging of PMNs over time. In one study, injured zebrafish larvae with GFP-labelled PMNs were exposed to compounds from 4 hours post-injury (a time point when high numbers of PMNs have been recruited to the wound site), to 12 hours post injury (when inflammation resolution was only partially complete in controls) ${ }^{190}$. Tanshinone IIA, which is derived from a Chinese medicinal herb, potently induced inflammation resolution by inducing PMN apoptosis and promoting reverse migration of PMNs. Tanshinone IIA also blocked pro-inflammatory signals in the zebrafish, and its effects were conserved in human PMNs, supporting the translational potential of this drug and this screening strategy.

A

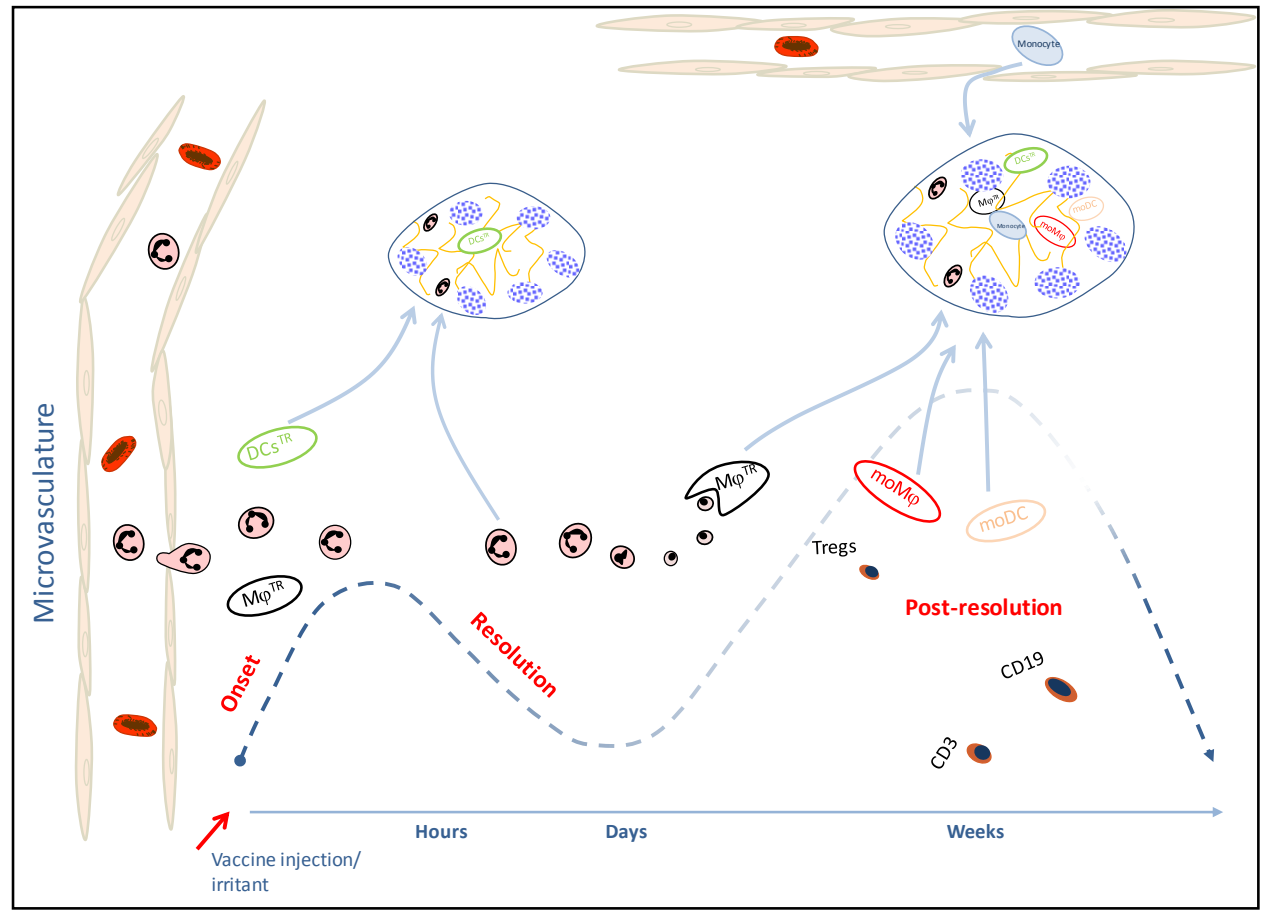

B 


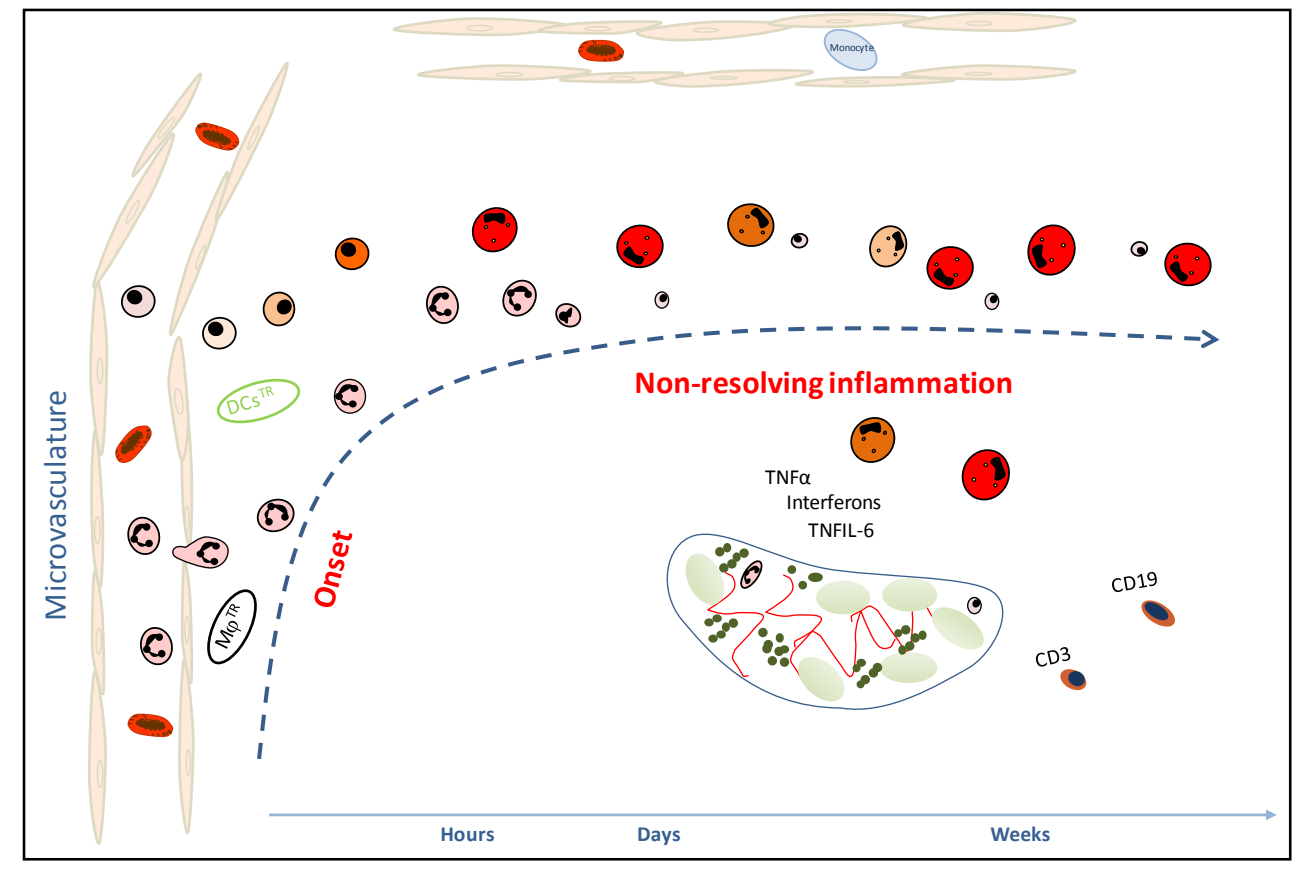

Figure 1. Resolution and adaptive immunity. We have recently shown ${ }^{4}$ that resolution is not the end of the immune response to infection or injury, but rather it acts as a bridge between innate and adaptive immunity, thereby adding a third phase to acute inflammation after acute and resolution - namely, post-resolution (a). Moreover, the idea that acute inflammation drives adaptive immuity also leads to an alternative explanation of the pathogenesis of some diseases that are driven by chronic inflammation (b). Specifically, diseases driven by 'inflammation-gone-wrong' may arise from incomplete resolution of the initial acute response that, in turn, does not fully engage an appropriate adaptive immune response that would otherwise lead to full resolution. Thus, although many early-phase Th-1-type cytokines have evolved to drive inflammation, their persistence may paradoxically derail resolution, resulting in impaired antigen clearance and the development of maladaptive immunity. 


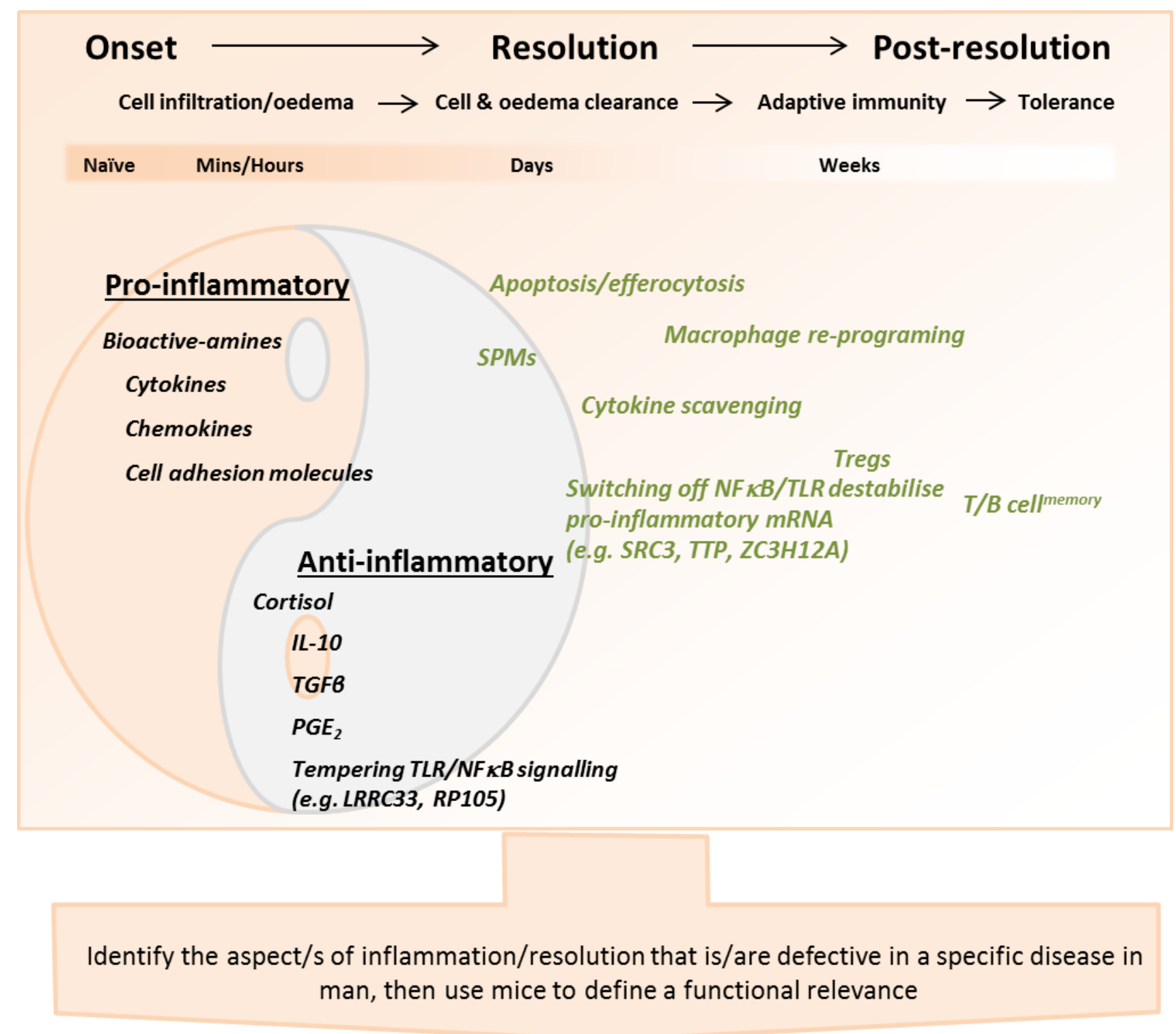

Rodents and experimental/translational medicine

Figure 2. Acute inflammation and its resolution. Responses to infection or injury are characterized by the early and sequential release of 'go signals', including proinflammatory cytokines and cell adhesion molecules, that collectively facilitate leukocyte migration into tissues. These events are counterbalanced by the concomitant release of 'stop signals', which serve to temper the severity of acute inflammation should it become too overexuberant and cause excessive tissue injury. These signals include IL-10, $\mathrm{PGE}_{2}$ and factors that control the magnitude of TLR and NFK-B signalling. Once the injurious agent is removed, pathways of resolution are activated, and include removal of the inciting stimulus and destabilization of proinflammatory mRNAs (via expression of SRC3, TTP and ZC3H12A). The latter, along with active pro-inflammatory mediator clearance (for example, through D6mediated scavenging) and leukocyte death followed by efferocytosis, leads to tissue resolution. These pro-resolution events were once thought to result in inflamed tissues reverting back to homeostasis; however, it is likely that they are a prelude to priming of adaptive immunity and also impact on the imprinting of innate immunity. 
A

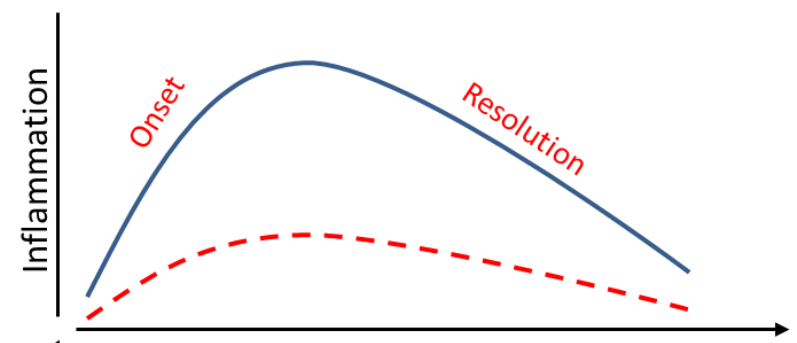

$\bigwedge_{\text {Anti-inflammatory }}$

B

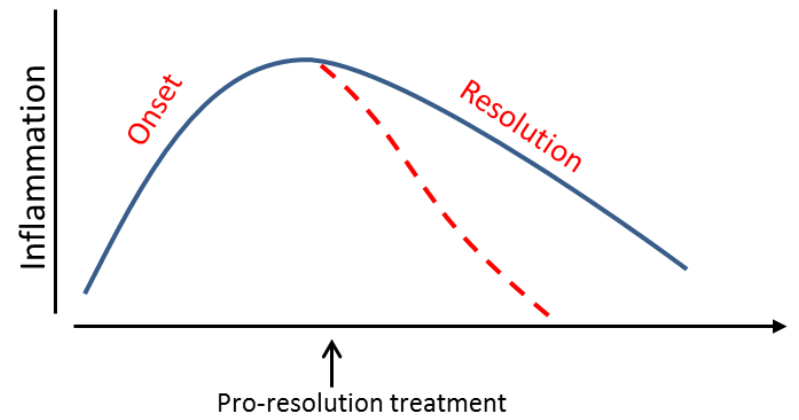

Figure 3. Anti-inflammation versus pro-resolution strategies. It is important to emphasise the difference between (a) anti-inflammation and (b) pro-resolution. The former describes the inhibition of factors that drive inflammation, including vasoactive amines, eicosanoids, cytokines, chemokines and cell adhesion molecules. Among the pharmacological tools developed to achieve this clinically are nonsteroidal antiinflammatory drugs and biologicals such as anti-TNF therapies. This form of intervention dampens inflammation from the onset with or without accelerated resolution. Pro-resolution, on the other hand, describes enhancing or promoting the factors essential for removal of the inciting stimulus as well as dampening proinflammatory signalling, followed by leukocyte clearance. It is envisioned that proresolution therapies will not necessarily affect onset but will accelerate resolution.

Table 1 | Pro-resolution mediators and their known functions in resolution.

\begin{tabular}{|c|c|c|c|c|c|c|c|c|c|}
\hline \multirow[b]{2}{*}{$\begin{array}{l}\text { Mediator or } \\
\text { effector }\end{array}$} & \multirow[b]{2}{*}{ Receptors } & \multicolumn{7}{|c|}{ Pro-resolution functions } & Refs \\
\hline & & 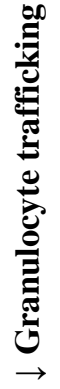 & 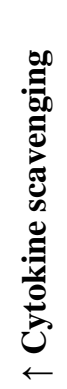 & 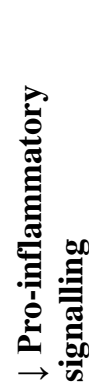 & 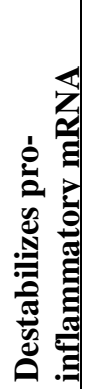 & 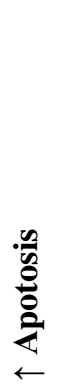 & 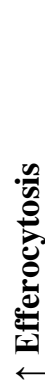 & 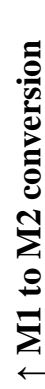 & \\
\hline
\end{tabular}




\begin{tabular}{|c|c|c|c|c|c|c|c|c|c|}
\hline Lipoxin $\mathrm{A}_{4} / \mathrm{B}_{4}$ & FPR2/ALX, GPR32 & + & + & & & & + & + & 191-194 \\
\hline Resolvin E1 & BLT1, Chem R23 & + & & + & & & + & + & $\begin{array}{l}195-197 \\
\end{array}$ \\
\hline Resolvin D1 & FPR2/ALX, GPR32 & + & & + & & & + & + & 198 \\
\hline Resolvin D2 & GPR18 & + & & & & & + & & 128 \\
\hline Maresins & Not known & + & & & & & + & & $199-202$ \\
\hline Protectin D1 & Not known & + & & & & + & & & 203,204 \\
\hline $\mathrm{PGD}_{2}$ & DP1, DP2 & + & & & & & & & $133,135,205-207$ \\
\hline $15-\operatorname{deoxy} \Delta^{12-14} \mathrm{PGJ}_{2}$ & PPAR $\gamma$ & + & & + & & & & & $133,135,207-209$ \\
\hline \multicolumn{10}{|l|}{ Protein } \\
\hline D6 & - & & + & & & & & & 210,211 \\
\hline $\begin{array}{l}\text { Annexin A1/Lipo- } \\
\text { cortin A1 }\end{array}$ & FPR2/ALX & + & & & & & + & + & $212-214$ \\
\hline Galectin 1 & $\begin{array}{l}\text { CD2, CD3, CD7, } \\
\text { CD43, CD45 }\end{array}$ & + & & & & + & + & & $215-217$ \\
\hline \multicolumn{10}{|l|}{ Galectin 9} \\
\hline Ac2-26 & FPR1, FPR2/ALX & + & & & & & + & & $212-214,218-220$ \\
\hline AlphaMSH & MC3R & + & & & & & + & & $221-224$ \\
\hline Chemerin 15 & ChemR23/CMKLR1 & + & & & & & + & & 225-227 \\
\hline \multicolumn{10}{|l|}{ Gaseous mediators } \\
\hline $\mathrm{CO}$ & - & & & & & & & & $228-230$ \\
\hline $\mathrm{H}_{2} \mathrm{~S}$ & - & + & & + & & + & & + & 147,149 \\
\hline \multicolumn{10}{|c|}{ Changes in intracellular signalling pathways } \\
\hline$\downarrow N F-\kappa B$ & - & & & & & + & & + & $74,80,231,232$ \\
\hline$\downarrow \mathrm{CDKs}$ & - & & & & & + & & & $90,130,233$ \\
\hline$\downarrow \mathrm{PI} 3 \mathrm{~K}$ & - & & & & & + & & & $73,76,80,234$ \\
\hline$\downarrow$ ERK1 or ERK2 & - & & & & & + & & & $84,200,235$ \\
\hline cAMP & - & & & & & + & + & + & $73,79,236-238$ \\
\hline \multicolumn{10}{|c|}{ Intracellular regulators } \\
\hline LRRC33 & - & & & + & & & & & 31 \\
\hline RP105 & - & & & + & & & & & 32 \\
\hline Cylindromatosis & - & & & + & & & & & 33 \\
\hline TTP & - & & & & + & & & & $38,40,42,239$ \\
\hline $\begin{array}{l}\text { MicroRNAs (miR- } \\
\text { 146, miR-21) }\end{array}$ & - & & & & + & & & & 55,60 \\
\hline \multicolumn{10}{|l|}{ Others } \\
\hline Adenosine & - & + & & & & & & + & 240,241 \\
\hline & & & & & & & & & \\
\hline
\end{tabular}

\section{Glossary}

\section{Non-phlogistic}

In an anti-inflammatory manner.

\section{Efferocytosis}

The removal of effete, mainly apoptotic, cells by professional phagocytes, especially macrophages. 


\section{Eicosanoids}

20-carbon structures that are derived from arachidonic acid. Examples include the prostaglandins and leukotrienes.

Adenine- or uridine-rich elements

(AREs). $3^{\prime}$ untranslated regions of mRNA that recruit destabilizing factors and translational silencers.

\section{microRNA}

(miRNA) A small non-coding RNA molecule containing about 21-22 nucleotides, which function by base-pairing with complementary sequences within mRNA molecules.

Polysensitization

A phenomenon in which sensitization to one allergen favours sensitization to other environmental allergens.

\section{Macrophage plasticity}

The ability of macrophages to alter or adapt their biological function commensurate with the inflammatory environment they inhabit.

\section{Granulocytes}

White blood cells that are characterized by granules in their cytoplasm. These include PMNs, eosinophils, basophils and mast cells.

\section{Stress granules}

Sites where untranslated mRNAs accumulate in cells that have been subjected to adverse environmental conditions.

\section{Exudate}

Cells and fluid that accumulate outside of blood vessels or an organ during inflammation.

\section{Acantholysis}

Pathological separation of the epidermis from the dermis.

\section{Zymosan}

A ligand found on the surface of fungi. It binds to TLR2 and Dectin receptors

\section{Co-inhibitory molecules}

Molecules belonging to the immunoglobulin superfamily or the tumour necrosis factor receptor superfamily that inhibit $\mathrm{T}$ cell receptor-mediated responses.

James N. Fullerton is a specialist registrar in clinical pharmacology at University College London Hospital, UK. Having studied at Oxford University (M.A.) and the University of 
Birmingham (UK, M.D.), he recently completed his $\mathrm{PhD}$ at University College London as a Wellcome Trust Research Training Fellow exploring the contribution of eicosanoids to immune dysfunction in the critically ill.

Derek W. Gilroy obtained his $\mathrm{PhD}$ from the William Harvey Research Institute, University of London in 1997. In 2010 he was promoted to Professor of Experimental Immunology at University College, where he is now Head of the Centre for Clinical Pharmacology. There, he has pioneered research examining the molecular and biochemical pathways that regulate the resolution of acute immune reactions.

\section{Key point summary}

1. Inflammation is a beneficial process, designed to contain and eradicate threats to the host organism. Dysregulation of the magnitude or duration of inflammation contributes to multiple pathologies.

2. Traditionally, drugs have been designed to reduce inflammation. Some such approaches - for example, non-steroidal anti-inflammatory drugs or proinflammatory cytokine ablation - achieve this by targeting factors that drive inflammation; others — for example, glucocorticoids — are directly antiinflammatory.

3. Active, specialized pathways bring about the resolution of inflammation. These involve discrete mediators and distinct cell phenotypes that act in a non-phlogistic manner to promote the clearance of inflammatory cells and a return to local tissue homeostasis.

4. Recent advances in our understanding of the central processes in the resolution of inflammation — including pro-inflammatory mediator catabolism, dampening of downstream signalling, apoptosis and efferocytosis of inflammatory cells, and their regulation - permit targeted pharmacological interventions to promote inflammatory resolution.

5. The development of drugs that promote or mimic the mode of action of endogenous pro-resolution pathways may afford a novel complementary, or potentially superior 
strategy, to traditional options — regulating inflammation and restoring function, not merely suppressing inflammation.

6. Recent discoveries suggest the innate immune response, and in particular its resolution, may modulate the subsequent development of adaptive immunity, and so may afford further therapeutic targets. Multiple challenges remain in developing human models of inflammatory resolution and in translating murine discoveries to date into drugs for man.

\section{ACKNOWLEDGEMENTS}

The authors wish to acknowledge the Wellcome Trust and the Medical Research Council for the financial support that has enabled the research that has contributed to this field.

\section{References}

1. Buckley, C.D., Gilroy, D.W. \& Serhan, C.N. Proresolving lipid mediators and mechanisms in the resolution of acute inflammation. Immunity 40, 315-27 (2014).

2. Buckley, C.D., Gilroy, D.W., Serhan, C.N., Stockinger, B. \& Tak, P.P. The resolution of inflammation. Nature reviews. Immunology 13, 59-66 (2013).

3. Gilroy, D.W. et al. Inducible cyclooxygenase-derived 15-deoxy(Delta)1214PGJ2 brings about acute inflammatory resolution in rat pleurisy by inducing neutrophil and macrophage apoptosis. Faseb J 17, 2269-71 (2003).

4. Newson, J. et al. Resolution of acute inflammation bridges the gap between innate and adaptive immunity. Blood 124, 1748-64 (2014).

5. Nakano, H. et al. Blood-derived inflammatory dendritic cells in lymph nodes stimulate acute T helper type 1 immune responses. Nat Immunol 10, 394-402 (2009).

6. Leon, B., Lopez-Bravo, M. \& Ardavin, C. Monocyte-derived dendritic cells formed at the infection site control the induction of protective $\mathrm{T}$ helper 1 responses against Leishmania. Immunity 26, 519-31 (2007).

7. Wakim, L.M. \& Bevan, M.J. Cross-dressed dendritic cells drive memory CD8+ T-cell activation after viral infection. Nature 471, 629-32 (2011).

8. Ersland, K., Wuthrich, M. \& Klein, B.S. Dynamic interplay among monocytederived, dermal, and resident lymph node dendritic cells during the generation of vaccine immunity to fungi. Cell Host Microbe 7, 474-87 (2010).

9. Uderhardt, S. et al. 12/15-Lipoxygenase Orchestrates the Clearance of Apoptotic Cells and Maintains Immunologic Tolerance. Immunity (2012).

10. Yona, S. et al. Fate mapping reveals origins and dynamics of monocytes and tissue macrophages under homeostasis. Immunity 38, 79-91 (2013).

11. Kool, M. et al. Alum adjuvant boosts adaptive immunity by inducing uric acid and activating inflammatory dendritic cells. J Exp Med 205, 869-82 (2008). 
12. Wilson, E.B. et al. Blockade of chronic type I interferon signaling to control persistent LCMV infection. Science 340, 202-7 (2013).

13. Teijaro, J.R. et al. Persistent LCMV infection is controlled by blockade of type I interferon signaling. Science 340, 207-11 (2013).

14. Gonzalez-Navajas, J.M., Lee, J., David, M. \& Raz, E. Immunomodulatory functions of type I interferons. Nat Rev Immunol 12, 125-35 (2012).

15. Boasso, A., Hardy, A.W., Anderson, S.A., Dolan, M.J. \& Shearer, G.M. HIVinduced type I interferon and tryptophan catabolism drive $\mathrm{T}$ cell dysfunction despite phenotypic activation. PloS one 3, e2961 (2008).

16. Cope, A.P. et al. Chronic tumor necrosis factor alters T cell responses by attenuating T cell receptor signaling. J Exp Med 185, 1573-84 (1997).

17. Serhan, C.N. et al. Resolution of inflammation: state of the art, definitions and terms. FASEB J 21, 325-32 (2007).

18. $\mathrm{Mi}$, S. et al. Blocking IL-17A promotes the resolution of pulmonary inflammation and fibrosis via TGF-beta1-dependent and -independent mechanisms. J Immunol 187, 3003-14 (2011).

19. Reddy, N.M., Potteti, H.R., Mariani, T.J., Biswal, S. \& Reddy, S.P. Conditional deletion of Nrf2 in airway epithelium exacerbates acute lung injury and impairs the resolution of inflammation. Am J Respir Cell Mol Biol 45, 1161-8 (2011).

20. Bynoe, M.S. et al. CD73 is critical for the resolution of murine colonic inflammation. J Biomed Biotechnol 2012, 260983 (2012).

21. Mavers, M. et al. Cyclin-dependent kinase inhibitor p21, via its C-terminal domain, is essential for resolution of murine inflammatory arthritis. Arthritis Rheum 64, 141-52 (2012).

22. Tomasini, R. et al. TAp73 is required for macrophage-mediated innate immunity and the resolution of inflammatory responses. Cell Death Differ 20, 293-301 (2013).

23. Wijbrandts, C.A. et al. Analysis of apoptosis in peripheral blood and synovial tissue very early after initiation of infliximab treatment in rheumatoid arthritis patients. Arthritis Rheum 58, 3330-9 (2008).

24. Thurlings, R.M. et al. Monocyte scintigraphy in rheumatoid arthritis: the dynamics of monocyte migration in immune-mediated inflammatory disease. PLoS One 4, e7865 (2009).

25. Chiang, N. et al. Infection regulates pro-resolving mediators that lower antibiotic requirements. Nature 484, 524-8 (2012).

26. Favas, C. \& Isenberg, D.A. B-cell-depletion therapy in SLE--what are the current prospects for its acceptance? Nat Rev Rheumatol 5, 711-6 (2009).

27. Isenberg, D.A. Rituximab-it was the best of times, it was the worst of times. Autoimmun Rev 11, 790-1 (2012).

28. Segal, A.W., Geisow, M., Garcia, R., Harper, A. \& Miller, R. The respiratory burst of phagocytic cells is associated with a rise in vacuolar pH. Nature 290, 406-9 (1981).

29. Pollock, J.D. et al. Mouse model of X-linked chronic granulomatous disease, an inherited defect in phagocyte superoxide production. Nat Genet 9, 202-9 (1995).

30. Stoecklin, G. \& Anderson, P. Posttranscriptional mechanisms regulating the inflammatory response. Adv Immunol 89, 1-37 (2006).

31. Liu, J. et al. Identification and characterization of a unique leucine-rich repeat protein (LRRC33) that inhibits Toll-like receptor-mediated NF-kappaB activation. Biochem Biophys Res Commun 434, 28-34 (2013). 
32. Divanovic, S. et al. Negative regulation of Toll-like receptor 4 signaling by the Toll-like receptor homolog RP105. Nat Immunol 6, 571-8 (2005).

33. Kovalenko, A. et al. The tumour suppressor CYLD negatively regulates NFkappaB signalling by deubiquitination. Nature 424, 801-5 (2003).

34. O'Brien, A.J. et al. Immunosuppression in acutely decompensated cirrhosis is mediated by prostaglandin E2. Nat Med 20, 518-23 (2014).

35. Degraaf, A.J., Zaslona, Z., Bourdonnay, E. \& Peters-Golden, M. Prostaglandin E2 reduces Toll-like receptor 4 expression in alveolar macrophages by inhibition of translation. Am J Respir Cell Mol Biol 51, 242-50 (2014).

36. Anderson, P. Post-transcriptional control of cytokine production. Nat Immunol 9, 353-9 (2008).

37. Carballo, E., Lai, W.S. \& Blackshear, P.J. Evidence that tristetraprolin is a physiological regulator of granulocyte-macrophage colony-stimulating factor messenger RNA deadenylation and stability. Blood 95, $1891-9$ (2000).

38. Ogilvie, R.L. et al. Tristetraprolin down-regulates IL-2 gene expression through AU-rich element-mediated mRNA decay. J Immunol 174, 953-61 (2005).

39. Sauer, I. et al. Interferons limit inflammatory responses by induction of tristetraprolin. Blood 107, 4790-7 (2006).

40. Linker, K. et al. Involvement of KSRP in the post-transcriptional regulation of human iNOS expression-complex interplay of KSRP with TTP and HuR. Nucleic Acids Res 33, 4813-27 (2005).

41. Phillips, K., Kedersha, N., Shen, L., Blackshear, P.J. \& Anderson, P. Arthritis suppressor genes TIA-1 and TTP dampen the expression of tumor necrosis factor alpha, cyclooxygenase 2, and inflammatory arthritis. Proc Natl Acad Sci U S A 101, 2011-6 (2004).

42. Ogilvie, R.L. et al. Tristetraprolin mediates interferon-gamma mRNA decay. J Biol Chem 284, 11216-23 (2009).

43. Carballo, E., Lai, W.S. \& Blackshear, P.J. Feedback inhibition of macrophage tumor necrosis factor-alpha production by tristetraprolin. Science 281, 1001-5 (1998).

44. Chen, Y.L. et al. Differential regulation of ARE-mediated TNFalpha and IL1 beta mRNA stability by lipopolysaccharide in RAW264.7 cells. Biochem Biophys Res Commun 346, 160-8 (2006).

45. von Roretz, C. \& Gallouzi, I.E. Decoding ARE-mediated decay: is microRNA part of the equation? J Cell Biol 181, 189-94 (2008).

46. Shyu, A.B., Wilkinson, M.F. \& van Hoof, A. Messenger RNA regulation: to translate or to degrade. EMBO J 27, 471-81 (2008).

47. Piecyk, M. et al. TIA-1 is a translational silencer that selectively regulates the expression of TNF-alpha. EMBO J 19, 4154-63 (2000).

48. Muhl, H. \& Pfeilschifter, J. Anti-inflammatory properties of pro-inflammatory interferon-gamma. Int Immunopharmacol 3, 1247-55 (2003).

49. Harvey, L.J. \& McArdle, H.J. Biomarkers of copper status: a brief update. The British journal of nutrition 99 Suppl 3, S10-3 (2008).

50. Sampath, P., Mazumder, B., Seshadri, V. \& Fox, P.L. Transcript-selective translational silencing by gamma interferon is directed by a novel structural element in the ceruloplasmin mRNA 3' untranslated region. $\mathrm{Mol} \mathrm{Cell} \mathrm{Biol} 23$, 1509-19 (2003). 
51. Mukhopadhyay, R., Jia, J., Arif, A., Ray, P.S. \& Fox, P.L. The GAIT system: a gatekeeper of inflammatory gene expression. Trends Biochem Sci 34, 324-31 (2009).

52. Vyas, K. et al. Genome-wide polysome profiling reveals an inflammationresponsive posttranscriptional operon in gamma interferon-activated monocytes. Mol Cell Biol 29, 458-70 (2009).

53. Liang, J. et al. A novel $\mathrm{CCCH}$-zinc finger protein family regulates proinflammatory activation of macrophages. J Biol Chem 283, 6337-46 (2008).

54. Matsushita, K. et al. Zc3h12a is an RNase essential for controlling immune responses by regulating mRNA decay. Nature 458, 1185-90 (2009).

55. Taganov, K.D., Boldin, M.P., Chang, K.J. \& Baltimore, D. NF-kappaBdependent induction of microRNA miR-146, an inhibitor targeted to signaling proteins of innate immune responses. Proc Natl Acad Sci U S A 103, 12481-6 (2006).

56. Perry, M.M. et al. Rapid changes in microRNA-146a expression negatively regulate the IL-1beta-induced inflammatory response in human lung alveolar epithelial cells. J Immunol 180, 5689-98 (2008).

57. Bhaumik, D. et al. MicroRNAs miR-146a/b negatively modulate the senescence-associated inflammatory mediators IL-6 and IL-8. Aging (Albany NY) 1, 402-11 (2009).

58. Jones, S.W. et al. The identification of differentially expressed microRNA in osteoarthritic tissue that modulate the production of TNF-alpha and MMP13. Osteoarthritis Cartilage 17, 464-72 (2009).

59. Nahid, M.A., Pauley, K.M., Satoh, M. \& Chan, E.K. miR-146a is critical for endotoxin-induced tolerance: IMPLICATION IN INNATE IMMUNITY. J Biol Chem 284, 34590-9 (2009).

60. Sheedy, F.J. et al. Negative regulation of TLR4 via targeting of the proinflammatory tumor suppressor PDCD4 by the microRNA miR-21. Nat Immunol 11, 141-7 (2010).

61. Schmidt, M.F. Drug target miRNAs: chances and challenges. Trends Biotechnol 32, 578-85 (2014).

62. Tili, E. et al. Modulation of miR-155 and miR-125b levels following lipopolysaccharide/TNF-alpha stimulation and their possible roles in regulating the response to endotoxin shock. J Immunol 179, 5082-9 (2007).

63. Bala, S. et al. Up-regulation of microRNA-155 in macrophages contributes to increased tumor necrosis factor \{alpha\} (TNF\{alpha\}) production via increased mRNA half-life in alcoholic liver disease. J Biol Chem 286, 1436-44 (2011).

64. Jones, M.R. et al. Zcchc11-dependent uridylation of microRNA directs cytokine expression. Nat Cell Biol 11, 1157-63 (2009).

65. Hansen, W.R., Keelan, J.A., Skinner, S.J. \& Mitchell, M.D. Key enzymes of prostaglandin biosynthesis and metabolism. Coordinate regulation of expression by cytokines in gestational tissues: a review. Prostaglandins Other Lipid Mediat 57, 243-57 (1999).

66. Hahn, E.L. et al. Prostaglandin E2 alterations during sepsis are partially mediated by endotoxin-induced inhibition of prostaglandin 15-

hydroxydehydrogenase. J Trauma 44, 777-81; discussion 781-2 (1998).

67. Nibbs, R.J. \& Graham, G.J. Immune regulation by atypical chemokine receptors. Nat Rev Immunol 13, 815-29 (2013). 
68. Ariel, A. et al. Apoptotic neutrophils and T cells sequester chemokines during immune response resolution through modulation of CCR5 expression. Nat Immunol 7, 1209-16 (2006).

69. Vanden Berghe, T., Linkermann, A., Jouan-Lanhouet, S., Walczak, H. \& Vandenabeele, P. Regulated necrosis: the expanding network of nonapoptotic cell death pathways. Nat Rev Mol Cell Biol 15, 135-47 (2014).

70. Remijsen, Q. et al. Dying for a cause: NETosis, mechanisms behind an antimicrobial cell death modality. Cell Death Differ 18, 581-8 (2011).

71. Savill, J. Apoptosis in resolution of inflammation. J Leukoc Biol 61, 375-80 (1997).

72. Cara, D.C., Negrao-Correa, D. \& Teixeira, M.M. Mechanisms underlying eosinophil trafficking and their relevance in vivo. Histol Histopathol 15, 899920 (2000).

73. Sousa, L.P. et al. Cyclic AMP enhances resolution of allergic pleurisy by promoting inflammatory cell apoptosis via inhibition of PI3K/Akt and NFkappaB. Biochem Pharmacol 78, 396-405 (2009).

74. Lawrence, T. \& Fong, C. The resolution of inflammation: anti-inflammatory roles for NF-kappaB. Int J Biochem Cell Biol 42, 519-23 (2010).

75. Song, G., Ouyang, G. \& Bao, S. The activation of Akt/PKB signaling pathway and cell survival. J Cell Mol Med 9, 59-71 (2005).

76. Rodrigues, D.H. et al. Absence of PI3Kgamma leads to increased leukocyte apoptosis and diminished severity of experimental autoimmune encephalomyelitis. J Neuroimmunol 222, 90-4 (2010).

77. Insel, P.A., Zhang, L., Murray, F., Yokouchi, H. \& Zambon, A.C. Cyclic AMP is both a pro-apoptotic and anti-apoptotic second messenger. Acta Physiol (Oxf) 204, 277-87 (2012).

78. Rossi, A.G. et al. Agents that elevate cAMP inhibit human neutrophil apoptosis. Biochem Biophys Res Commun 217, 892-9 (1995).

79. Rossi, A.G. et al. Regulation of macrophage phagocytosis of apoptotic cells by cAMP. J Immunol 160, 3562-8 (1998).

80. Sousa, L.P. et al. PDE4 inhibition drives resolution of neutrophilic inflammation by inducing apoptosis in a PKA-PI3K/Akt-dependent and NFkappaB-independent manner. J Leukoc Biol 87, 895-904 (2010).

81. Bystrom, J. et al. Resolution-phase macrophages possess a unique inflammatory phenotype that is controlled by cAMP. Blood 112, 4117-27 (2008).

82. Junttila, M.R., Li, S.P. \& Westermarck, J. Phosphatase-mediated crosstalk between MAPK signaling pathways in the regulation of cell survival. FASEB $J$ 22, 954-65 (2008).

83. Chapman, M.S. \& Miner, J.N. Novel mitogen-activated protein kinase kinase inhibitors. Expert Opin Investig Drugs 20, 209-20 (2011).

84. Sawatzky, D.A., Willoughby, D.A., Colville-Nash, P.R. \& Rossi, A.G. The involvement of the apoptosis-modulating proteins ERK 1/2, $\mathrm{Bcl}-\mathrm{xL}$ and $\mathrm{Bax}$ in the resolution of acute inflammation in vivo. Am J Pathol 168, 33-41 (2006).

85. Langereis, J.D., Raaijmakers, H.A., Ulfman, L.H. \& Koenderman, L. Abrogation of NF-kappaB signaling in human neutrophils induces neutrophil survival through sustained p38-MAPK activation. J Leukoc Biol 88, 655-64 (2010). 
86. Allaeys, I., Gymninova, I., Canet-Jourdan, C. \& Poubelle, P.E. IL-32gamma delays spontaneous apoptosis of human neutrophils through MCL-1, regulated primarily by the p38 MAPK pathway. PloS one 9, e109256 (2014).

87. Perdiguero, E., Kharraz, Y., Serrano, A.L. \& Munoz-Canoves, P. MKP-1 coordinates ordered macrophage-phenotype transitions essential for stem cell-dependent tissue repair. Cell Cycle 11, 877-86 (2012).

88. Chung, E.Y. et al. Interleukin-10 expression in macrophages during phagocytosis of apoptotic cells is mediated by homeodomain proteins $\mathrm{Pbx} 1$ and Prep-1. Immunity 27, 952-64 (2007).

89. Leitch, A.E. et al. Cyclin-dependent kinases 7 and 9 specifically regulate neutrophil transcription and their inhibition drives apoptosis to promote resolution of inflammation. Cell Death Differ 19, 1950-61 (2012).

90. Rossi, A.G. et al. Cyclin-dependent kinase inhibitors enhance the resolution of inflammation by promoting inflammatory cell apoptosis. Nat Med 12, 1056-64 (2006).

91. Leitch, A.E. et al. The cyclin-dependent kinase inhibitor R-roscovitine downregulates $\mathrm{Mcl}-1$ to override pro-inflammatory signalling and drive neutrophil apoptosis. Eur J Immunol 40, 1127-38 (2010).

92. Alessandri, A.L. et al. Induction of eosinophil apoptosis by the cyclindependent kinase inhibitor AT7519 promotes the resolution of eosinophildominant allergic inflammation. PloS one 6, e25683 (2011).

93. Shao, W.H. \& Cohen, P.L. Disturbances of apoptotic cell clearance in systemic lupus erythematosus. Arthritis Res Ther 13, 202 (2011).

94. Brown, J.R., Goldblatt, D., Buddle, J., Morton, L. \& Thrasher, A.J. Diminished production of anti-inflammatory mediators during neutrophil apoptosis and macrophage phagocytosis in chronic granulomatous disease (CGD). J Leukoc Biol 73, 591-9 (2003).

95. Elliott, M.R. et al. Nucleotides released by apoptotic cells act as a find-me signal to promote phagocytic clearance. Nature 461, 282-6 (2009).

96. Lauber, K. et al. Apoptotic cells induce migration of phagocytes via caspase3-mediated release of a lipid attraction signal. Cell 113, 717-30 (2003).

97. Gude, D.R. et al. Apoptosis induces expression of sphingosine kinase 1 to release sphingosine-1-phosphate as a "come-and-get-me" signal. FASEB J 22, 2629-38 (2008).

98. Knies, U.E. et al. Regulation of endothelial monocyte-activating polypeptide II release by apoptosis. Proc Natl Acad Sci U S A 95, $12322-7$ (1998).

99. Horino, K. et al. A monocyte chemotactic factor, S19 ribosomal protein dimer, in phagocytic clearance of apoptotic cells. Laboratory investigation; a journal of technical methods and pathology 78, 603-17 (1998).

100. Savill, J., Hogg, N., Ren, Y. \& Haslett, C. Thrombospondin cooperates with CD36 and the vitronectin receptor in macrophage recognition of neutrophils undergoing apoptosis. J Clin Invest 90, 1513-22 (1992).

101. Schwab, J.M., Chiang, N., Arita, M. \& Serhan, C.N. Resolvin E1 and protectin D1 activate inflammation-resolution programmes. Nature 447, 869-74 (2007).

102. Vago, J.P. et al. Annexin A1 modulates natural and glucocorticoid-induced resolution of inflammation by enhancing neutrophil apoptosis. J Leukoc Biol 92, 249-58 (2012).

103. Brown, S. et al. Apoptosis disables CD31-mediated cell detachment from phagocytes promoting binding and engulfment. Nature 418, 200-3 (2002). 
104. Gardai, S.J. et al. Cell-surface calreticulin initiates clearance of viable or apoptotic cells through trans-activation of LRP on the phagocyte. Cell 123, 321-34 (2005).

105. Fadok, V.A., Bratton, D.L. \& Henson, P.M. Phagocyte receptors for apoptotic cells: recognition, uptake, and consequences. J Clin Invest 108, 957-62 (2001).

106. Gregory, C.D. \& Pound, J.D. Microenvironmental influences of apoptosis in vivo and in vitro. Apoptosis 15, 1029-49 (2010).

107. Park, D. et al. BAl1 is an engulfment receptor for apoptotic cells upstream of the ELMO/Dock180/Rac module. Nature 450, 430-4 (2007).

108. Devitt, A. et al. Human CD14 mediates recognition and phagocytosis of apoptotic cells. Nature 392, 505-9 (1998).

109. Fadok, V.A. \& Henson, P.M. Apoptosis: giving phosphatidylserine recognition an assist--with a twist. Curr Biol 13, R655-7 (2003).

110. Bystrom, J., Wynn, T.A., Domachowske, J.B. \& Rosenberg, H.F. Gene microarray analysis reveals interleukin-5-dependent transcriptional targets in mouse bone marrow. Blood 103, 868-77 (2004).

111. Bellingan, G.J. et al. Adhesion molecule-dependent mechanisms regulate the rate of macrophage clearance during the resolution of peritoneal inflammation. J Exp Med 196, 1515-21 (2002).

112. Ariel, A. \& Serhan, C.N. New Lives Given by Cell Death: Macrophage Differentiation Following Their Encounter with Apoptotic Leukocytes during the Resolution of Inflammation. Front Immunol 3, 4 (2012).

113. Schif-Zuck, S. et al. Saturated-efferocytosis generates pro-resolving CD11b low macrophages: modulation by resolvins and glucocorticoids. Eur $J$ Immunol 41, 366-79 (2011).

114. Fadok, V.A., Warner, M.L., Bratton, D.L. \& Henson, P.M. CD36 is required for phagocytosis of apoptotic cells by human macrophages that use either a phosphatidylserine receptor or the vitronectin receptor (alpha $v$ beta 3 ). $J$ Immunol 161, 6250-7 (1998).

115. Fadok, V.A. \& Henson, P.M. Apoptosis: getting rid of the bodies. Curr Bio/ 8 , R693-5 (1998).

116. Fadok, V.A. et al. Macrophages that have ingested apoptotic cells in vitro inhibit proinflammatory cytokine production through autocrine/paracrine mechanisms involving TGF-beta, PGE2, and PAF. J Clin Invest 101, 890-8 (1998).

117. Serhan, C.N. et al. Macrophage proresolving mediator maresin 1 stimulates tissue regeneration and controls pain. FASEB J 26, 1755-65 (2012).

118. Medeiros, A.I., Serezani, C.H., Lee, S.P. \& Peters-Golden, M. Efferocytosis impairs pulmonary macrophage and lung antibacterial function via PGE2/EP2 signaling. J Exp Med 206, 61-8 (2009).

119. van Rijt, L.S. et al. Persistent activation of dendritic cells after resolution of allergic airway inflammation breaks tolerance to inhaled allergens in mice. Am J Respir Crit Care Med 184, 303-11 (2011).

120. Rosas, M. et al. The transcription factor Gata6 links tissue macrophage phenotype and proliferative renewal. Science 344, 645-8 (2014).

121. Okabe, Y. \& Medzhitov, R. Tissue-specific signals control reversible program of localization and functional polarization of macrophages. Cell 157, 832-44 (2014). 
122. Ramachandran, P. et al. Differential Ly-6C expression identifies the recruited macrophage phenotype, which orchestrates the regression of murine liver fibrosis. Proc Natl Acad Sci U S A 109, E3186-95 (2012).

123. Anders, H.J. \& Ryu, M. Renal microenvironments and macrophage phenotypes determine progression or resolution of renal inflammation and fibrosis. Kidney international 80, 915-25 (2011).

124. Arnold, L. et al. Inflammatory monocytes recruited after skeletal muscle injury switch into antiinflammatory macrophages to support myogenesis. J Exp Med 204, 1057-69 (2007).

125. Mounier, R. et al. AMPKalpha1 regulates macrophage skewing at the time of resolution of inflammation during skeletal muscle regeneration. Cell Metab 18, 251-64 (2013).

126. Bannenberg, G.L. et al. Molecular circuits of resolution: formation and actions of resolvins and protectins. J Immunol 174, 4345-55 (2005).

127. Zmijewski, J.W. et al. Mitochondrial respiratory complex I regulates neutrophil activation and severity of lung injury. Am J Respir Crit Care Med 178, 168-79 (2008).

128. Serhan, C.N. Pro-resolving lipid mediators are leads for resolution physiology. Nature 510, 92-101 (2014).

129. Alessandri, A.L. et al. Resolution of inflammation: mechanisms and opportunity for drug development. Pharmacology \& therapeutics 139, 189-212 (2013).

130. Poon, I.K., Lucas, C.D., Rossi, A.G. \& Ravichandran, K.S. Apoptotic cell clearance: basic biology and therapeutic potential. Nat Rev Immunol 14, 16680 (2014).

131. Koedel, U. et al. Apoptosis is essential for neutrophil functional shutdown and determines tissue damage in experimental pneumococcal meningitis. PLoS Pathog 5, e1000461 (2009).

132. Cash, J.L., Norling, L.V. \& Perretti, M. Resolution of inflammation: targeting GPCRs that interact with lipids and peptides. Drug Discov Today 19, 1186-92 (2014).

133. Gilroy, D.W. et al. Inducible cyclooxygenase may have anti-inflammatory properties. Nat Med 5, 698-701 (1999).

134. Rajakariar, R. et al. Hematopoietic prostaglandin D2 synthase controls the onset and resolution of acute inflammation through PGD2 and 15deoxyDelta12 14 PGJ2. Proc Natl Acad Sci U S A 104, 20979-84 (2007).

135. Trivedi, S.G. et al. Essential role for hematopoietic prostaglandin D2 synthase in the control of delayed type hypersensitivity. Proc Natl Acad Sci U S A 103, 5179-84 (2006).

136. Chan, M.M. \& Moore, A.R. Resolution of inflammation in murine autoimmune arthritis is disrupted by cyclooxygenase-2 inhibition and restored by prostaglandin E2-mediated lipoxin A4 production. J Immunol 184, 6418-26 (2010).

137. Straus, D.S. \& Glass, C.K. Anti-inflammatory actions of PPAR ligands: new insights on cellular and molecular mechanisms. Trends Immunol 28, 551-8 (2007).

138. Rossi, A. et al. Anti-inflammatory cyclopentenone prostaglandins are direct inhibitors of IkappaB kinase. Nature 403, 103-8 (2000).

139. Kim, W.J., Kim, J.H. \& Jang, S.K. Anti-inflammatory lipid mediator 15d-PGJ2 inhibits translation through inactivation of elF4A. EMBO J 26, 5020-32 (2007). 
140. Anderson, P. \& Kedersha, N. Stress granules: the Tao of RNA triage. Trends Biochem Sci 33, 141-50 (2008).

141. Recchiuti, A., Krishnamoorthy, S., Fredman, G., Chiang, N. \& Serhan, C.N. MicroRNAs in resolution of acute inflammation: identification of novel resolvin D1-miRNA circuits. FASEB J 25, 544-60 (2011).

142. Krishnamoorthy, S., Recchiuti, A., Chiang, N., Fredman, G. \& Serhan, C.N. Resolvin D1 receptor stereoselectivity and regulation of inflammation and proresolving microRNAs. Am J Pathol 180, 2018-27 (2012).

143. Braley-Mullen, H. \& Sharp, G.C. Adoptive transfer murine model of granulomatous experimental autoimmune thyroiditis. Int Rev Immunol 19, 535-55 (2000).

144. Fang, Y., Sharp, G.C., Yagita, H. \& Braley-Mullen, H. A critical role for TRAIL in resolution of granulomatous experimental autoimmune thyroiditis. J Pathol 216, 505-13 (2008).

145. Fang, Y., Sharp, G.C. \& Braley-Mullen, H. Interleukin-10 promotes resolution of granulomatous experimental autoimmune thyroiditis. Am J Pathol 172, 1591-602 (2008).

146. McGrath, E.E. et al. TNF-related apoptosis-inducing ligand (TRAIL) regulates inflammatory neutrophil apoptosis and enhances resolution of inflammation. $J$ Leukoc Biol 90, 855-65 (2011).

147. Gemici, B. et al. HS-releasing drugs: anti-inflammatory, cytoprotective and chemopreventative potential. Nitric Oxide (2014).

148. Dufton, N., Natividad, J., Verdu, E.F. \& Wallace, J.L. Hydrogen sulfide and resolution of acute inflammation: A comparative study utilizing a novel fluorescent probe. Sci Rep 2, 499 (2012).

149. Wallace, J.L. \& Wang, R. Hydrogen sulfide-based therapeutics: exploiting a unique but ubiquitous gasotransmitter. Nat Rev Drug Discov 14, 329-45 (2015).

150. Montero-Melendez, T. ACTH: The forgotten therapy. Semin Immunol 27, 21626 (2015).

151. Matzelle, M.M. et al. Resolution of inflammation induces osteoblast function and regulates the Wnt signaling pathway. Arthritis Rheum 64, 1540-50 (2012).

152. Chiang, N., Dalli, J., Colas, R.A. \& Serhan, C.N. Identification of resolvin D2 receptor mediating resolution of infections and organ protection. $J$ Exp Med 212, 1203-17 (2015).

153. Cahill, R.N., Frost, H. \& Trnka, Z. The effects of antigen on the migration of recirculating lymphocytes through single lymph nodes. $J$ Exp Med 143, 87088 (1976).

154. Hopkins, J., McConnell, I. \& Pearson, J.D. Lymphocyte traffic through antigenstimulated lymph nodes. II. Role of Prostaglandin E2 as a mediator of cell shutdown. Immunology 42, 225-31 (1981).

155. McConnell, I. \& Hopkins, J. Lymphocyte traffic through antigen-stimulated lymph nodes. I. Complement activation within lymph nodes initiates cell shutdown. Immunology 42, 217-23 (1981).

156. Johnston, M.G., Hay, J.B. \& Movat, H.Z. Kinetics of prostaglandin production in various inflammatory lesions, measured in draining lymph. Am J Pathol 95, 225-38 (1979).

157. Yang, C.W. \& Unanue, E.R. Neutrophils control the magnitude and spread of the immune response in a thromboxane A2-mediated process. J Exp Med 210, 375-87 (2013). 
158. Yao, C. et al. Prostaglandin $\mathrm{E}(2)$ promotes Th1 differentiation via synergistic amplification of IL-12 signalling by CAMP and PI3-kinase. Nat Commun 4, 1685 (2013).

159. Mitroulis, I., Kourtzelis, I., Kambas, K., Chrysanthopoulou, A. \& Ritis, K. Evidence for the involvement of mTOR inhibition and basal autophagy in familial Mediterranean fever phenotype. Hum Immunol 72, 135-8 (2011).

160. Deretic, V., Saitoh, T. \& Akira, S. Autophagy in infection, inflammation and immunity. Nat Rev Immunol 13, 722-37 (2013).

161. Amulic, B. \& Hayes, G. Neutrophil extracellular traps. Curr Biol 21, R297-8 (2011).

162. Mitroulis, I. et al. Neutrophil extracellular trap formation is associated with IL1 beta and autophagy-related signaling in gout. PloS one 6, e29318 (2011).

163. Mocarski, E.S., Kaiser, W.J., Livingston-Rosanoff, D., Upton, J.W. \& DaleyBauer, L.P. True grit: programmed necrosis in antiviral host defense, inflammation, and immunogenicity. J Immunol 192, 2019-26 (2014).

164. Berger, S.B. et al. Cutting Edge: RIP1 kinase activity is dispensable for normal development but is a key regulator of inflammation in SHARPINdeficient mice. J Immunol 192, 5476-80 (2014).

165. Prince, L.R. et al. Staphylococcus aureus induces eosinophil cell death mediated by alpha-hemolysin. PloS one 7, e31506 (2012).

166. Feoktistova, M. et al. clAPs block Ripoptosome formation, a RIP1/caspase-8 containing intracellular cell death complex differentially regulated by cFLIP isoforms. Mol Cell 43, 449-63 (2011).

167. Chan, F.K. Fueling the flames: Mammalian programmed necrosis in inflammatory diseases. Cold Spring Harb Perspect Biol 4 (2012).

168. Miles, K. et al. Dying and necrotic neutrophils are anti-inflammatory secondary to the release of alpha-defensins. J Immunol 183, 2122-32 (2009).

169. Blume, K.E. et al. Cell surface externalization of annexin A1 as a failsafe mechanism preventing inflammatory responses during secondary necrosis. $J$ Immunol 183, 8138-47 (2009).

170. Menzies, F.M., Moreau, K. \& Rubinsztein, D.C. Protein misfolding disorders and macroautophagy. Current opinion in cell biology 23, 190-7 (2011).

171. Mihalache, C.C. et al. Inflammation-associated autophagy-related programmed necrotic death of human neutrophils characterized by organelle fusion events. J Immunol 186, 6532-42 (2011).

172. Schauer, C. et al. Aggregated neutrophil extracellular traps limit inflammation by degrading cytokines and chemokines. Nat Med 20, 511-7 (2014).

173. Seok, J. et al. Genomic responses in mouse models poorly mimic human inflammatory diseases. Proc Natl Acad Sci U S A 110, 3507-12 (2013).

174. Takao, K. \& Miyakawa, T. Genomic responses in mouse models greatly mimic human inflammatory diseases. Proc Natl Acad Sci U S A 112, 1167-72 (2015).

175. Shay, T., Lederer, J.A. \& Benoist, C. Genomic responses to inflammation in mouse models mimic humans: We concur, apples to oranges comparisons won't do. Proc Natl Acad Sci U S A 112, E346 (2015).

176. Libby, P., Tabas, I., Fredman, G. \& Fisher, E.A. Inflammation and its resolution as determinants of acute coronary syndromes. Circ Res 114, 186779 (2014).

177. Nicholls, S.J. et al. Effect of two intensive statin regimens on progression of coronary disease. N Engl J Med 365, 2078-87 (2011). 
178. Swirski, F.K. et al. Ly-6Chi monocytes dominate hypercholesterolemiaassociated monocytosis and give rise to macrophages in atheromata. $J$ Clin Invest 117, 195-205 (2007).

179. Drechsler, M., Megens, R.T., van Zandvoort, M., Weber, C. \& Soehnlein, O. Hyperlipidemia-triggered neutrophilia promotes early atherosclerosis. Circulation 122, 1837-45 (2010).

180. Parathath, S. et al. Diabetes adversely affects macrophages during atherosclerotic plaque regression in mice. Diabetes 60, 1759-69 (2011).

181. Day, R.M., Harbord, M., Forbes, A. \& Segal, A.W. Cantharidin blisters: a technique for investigating leukocyte trafficking and cytokine production at sites of inflammation in humans. J Immunol Methods 257, 213-20 (2001).

182. Evans, B.J., Haskard, D.O., Sempowksi, G. \& Landis, R.C. Evolution of the Macrophage CD163 Phenotype and Cytokine Profiles in a Human Model of Resolving Inflammation. Int J Inflam 2013, 780502 (2013).

183. Jenner, W. et al. Characterisation of leukocytes in a human skin blister model of acute inflammation and resolution. PLoS One 9, e89375 (2014).

184. Jenner, W.J. \& Gilroy, D.W. Assessment of leukocyte trafficking in humans using the cantharidin blister model. JRSM Cardiovasc Dis 1 (2012).

185. Basran, A. et al. Roles of neutrophils in the regulation of the extent of human inflammation through delivery of IL-1 and clearance of chemokines. J Leukoc Biol 93, 7-19 (2013).

186. Brittan, M. et al. A novel subpopulation of monocyte-like cells in the human lung after lipopolysaccharide inhalation. Eur Respir J 40, 206-14 (2012).

187. Vukmanovic-Stejic, M., Rustin, M.H., Nikolich-Zugich, J. \& Akbar, A.N. Immune responses in the skin in old age. Curr Opin Immunol 23, 525-31 (2011).

188. High, K.P., Akbar, A.N. \& Nikolich-Zugich, J. Translational research in immune senescence: assessing the relevance of current models. Semin Immunol 24, 373-82 (2012).

189. Gilroy, D.W., Lawrence, T., Perretti, M. \& Rossi, A.G. Inflammatory resolution: new opportunities for drug discovery. Nat Rev Drug Discov 3, 401-16 (2004).

190. Robertson, A.L. et al. A zebrafish compound screen reveals modulation of neutrophil reverse migration as an anti-inflammatory mechanism. Sci Trans/ Med 6, 225ra29 (2014).

191. Back, M. et al. Update on leukotriene, lipoxin and oxoeicosanoid receptors: IUPHAR Review 7. Br J Pharmacol 171, 3551-74 (2014).

192. Machado, F.S. \& Aliberti, J. Role of lipoxin in the modulation of immune response during infection. Int Immunopharmacol 8, 1316-9 (2008).

193. Romano, M. Lipoxin and aspirin-triggered lipoxins. ScientificWorldJournal 10, 1048-64 (2010).

194. Romano, M., Recchia, I. \& Recchiuti, A. Lipoxin receptors. ScientificWorldJournal 7, 1393-412 (2007).

195. Gyurko, R. \& Van Dyke, T.E. The role of polyunsaturated omega-3 fatty acid eicosapentaenoic acid-derived resolvin E1 (RvE1) in bone preservation. Crit Rev Immunol 34, 347-57 (2014).

196. Hisada, T., Ishizuka, T., Aoki, H. \& Mori, M. Resolvin E1 as a novel agent for the treatment of asthma. Expert Opin Ther Targets 13, 513-22 (2009).

197. Seki, H., Tani, Y. \& Arita, M. Omega-3 PUFA derived anti-inflammatory lipid mediator resolvin E1. Prostaglandins Other Lipid Mediat 89, 126-30 (2009). 
198. Recchiuti, A. Resolvin D1 and its GPCRs in resolution circuits of inflammation. Prostaglandins Other Lipid Mediat 107, 64-76 (2013).

199. Chatterjee, A. et al. The pro-resolving lipid mediator maresin 1 (MaR1) attenuates inflammatory signaling pathways in vascular smooth muscle and endothelial cells. PLoS One 9, e113480 (2014).

200. Gong, J. et al. Maresin 1 Prevents Lipopolysaccharide-Induced Neutrophil Survival and Accelerates Resolution of Acute Lung Injury. Shock 44, 371-80 (2015).

201. Sasaki, K., Urabe, D., Arai, H., Arita, M. \& Inoue, M. Total synthesis and bioactivities of two proposed structures of maresin. Chem Asian J 6, 534-43 (2011).

202. Wang, C.W. et al. Maresin 1 Biosynthesis and Pro-resolving Anti-Infective Functions with Human Localized Aggressive Periodontitis Leukocytes. Infect Immun (2015).

203. Lagarde, M. et al. Docosahexaenoic acid, protectin synthesis: relevance against atherothrombogenesis. Proc Nutr Soc 73, 186-9 (2014).

204. Weylandt, K.H., Chiu, C.Y., Gomolka, B., Waechter, S.F. \& Wiedenmann, B. Omega-3 fatty acids and their lipid mediators: towards an understanding of resolvin and protectin formation. Prostaglandins Other Lipid Mediat 97, 73-82 (2012).

205. Pettipher, R., Hansel, T.T. \& Armer, R. Antagonism of the prostaglandin D2 receptors DP1 and CRTH2 as an approach to treat allergic diseases. Nat Rev Drug Discov 6, 313-25 (2007).

206. Uehara, Y. [Prostaglandin D2]. Nihon Rinsho 57 Suppl, 728-31 (1999).

207. Rajakariar, R. et al. Hematopoietic prostaglandin D2 synthase controls the onset and resolution of acute inflammation through PGD2 and 15deoxyDelta12 14 PGJ2. Proceedings of the National Academy of Sciences of the United States of America 104, 20979-84 (2007).

208. Uchida, K. \& Shibata, T. 15-Deoxy-Delta(12,14)-prostaglandin J2: an electrophilic trigger of cellular responses. Chem Res Toxicol 21, 138-44 (2008).

209. Clay, C.E. et al. 15-deoxy-Delta(12,14)PGJ(2) induces diverse biological responses via PPARgamma activation in cancer cells. Prostaglandins Other Lipid Mediat 62, 23-32 (2000).

210. Graham, G.J. D6/Ackr2. Front Immunol 6, 280 (2015).

211. Graham, G.J. \& Locati, M. Regulation of the immune and inflammatory responses by the 'atypical' chemokine receptor D6. J Pathol 229, 168-75 (2013).

212. Chen, L., Lv, F. \& Pei, L. Annexin 1: a glucocorticoid-inducible protein that modulates inflammatory pain. Eur J Pain 18, 338-47 (2014).

213. Perretti, M. \& D'Acquisto, F. Annexin A1 and glucocorticoids as effectors of the resolution of inflammation. Nat Rev Immunol 9, 62-70 (2009).

214. Yazid, S., Norling, L.V. \& Flower, R.J. Anti-inflammatory drugs, eicosanoids and the annexin A1/FPR2 anti-inflammatory system. Prostaglandins Other Lipid Mediat 98, 94-100 (2012).

215. Henderson, N.C. \& Sethi, T. The regulation of inflammation by galectin-3. Immunol Rev 230, 160-71 (2009).

216. Rabinovich, G.A. \& Toscano, M.A. Turning 'sweet' on immunity: galectinglycan interactions in immune tolerance and inflammation. Nat Rev Immunol 9, 338-52 (2009). 
217. Elola, M.T., Chiesa, M.E., Alberti, A.F., Mordoh, J. \& Fink, N.E. Galectin-1 receptors in different cell types. J Biomed Sci 12, 13-29 (2005).

218. Gavins, F.N., Leoni, G. \& Getting, S.J. Annexin 1 and melanocortin peptide therapy for protection against ischaemic-reperfusion damage in the heart. ScientificWorldJournal 6, 1008-23 (2006).

219. Perretti, M. Lipocortin 1 and chemokine modulation of granulocyte and monocyte accumulation in experimental inflammation. Gen Pharmacol 31, 545-52 (1998).

220. Qin, C. et al. Cardioprotective potential of annexin-A1 mimetics in myocardial infarction. Pharmacol Ther 148, 47-65 (2015).

221. Brzoska, T., Bohm, M., Lugering, A., Loser, K. \& Luger, T.A. Terminal signal: anti-inflammatory effects of alpha-melanocyte-stimulating hormone related peptides beyond the pharmacophore. Adv Exp Med Biol 681, 107-16 (2010).

222. D'Agostino, G. \& Diano, S. Alpha-melanocyte stimulating hormone: production and degradation. J Mol Med (Berl) 88, 1195-201 (2010).

223. Dores, R.M. Adrenocorticotropic hormone, melanocyte-stimulating hormone, and the melanocortin receptors: revisiting the work of Robert Schwyzer: a thirty-year retrospective. Ann N Y Acad Sci 1163, 93-100 (2009).

224. Singh, M. \& Mukhopadhyay, K. Alpha-melanocyte stimulating hormone: an emerging anti-inflammatory antimicrobial peptide. Biomed Res Int 2014, 874610 (2014).

225. Bondue, B., Wittamer, V. \& Parmentier, M. Chemerin and its receptors in leukocyte trafficking, inflammation and metabolism. Cytokine Growth Factor Rev 22, 331-8 (2011).

226. Mariani, F. \& Roncucci, L. Chemerin/chemR23 axis in inflammation onset and resolution. Inflamm Res 64, 85-95 (2015).

227. Zabel, B.A. et al. Chemerin regulation and role in host defense. Am J Clin Exp Immunol 3, 1-19 (2014).

228. Chung, H.T., Choi, B.M., Kwon, Y.G. \& Kim, Y.M. Interactive relations between nitric oxide (NO) and carbon monoxide (CO): heme oxygenase-1/CO pathway is a key modulator in NO-mediated antiapoptosis and antiinflammation. Methods Enzymol 441, 329-38 (2008).

229. Li, L., Hsu, A. \& Moore, P.K. Actions and interactions of nitric oxide, carbon monoxide and hydrogen sulphide in the cardiovascular system and in inflammation--a tale of three gases! Pharmacol Ther 123, 386-400 (2009).

230. Ryter, S.W. \& Choi, A.M. Targeting heme oxygenase-1 and carbon monoxide for therapeutic modulation of inflammation. Transl Res 167, 7-34 (2016).

231. Fong, C.H. et al. An antiinflammatory role for IKKbeta through the inhibition of "classical" macrophage activation. J Exp Med 205, 1269-76 (2008).

232. Liu, J. et al. PI3K is required for the physical interaction and functional inhibition of NF-kappaB by beta-catenin in colorectal cancer cells. Biochem Biophys Res Commun 434, 760-6 (2013).

233. Rossi, A.G. L13. Apoptosis, apoptotic cell clearance and resolution of inflammation. Presse Med 42, 536-7 (2013).

234. Geering, B., Gurzeler, U., Federzoni, E., Kaufmann, T. \& Simon, H.U. A novel TNFR1-triggered apoptosis pathway mediated by class IA PI3Ks in neutrophils. Blood 117, 5953-62 (2011).

235. El Kebir, D., Gjorstrup, P. \& Filep, J.G. Resolvin E1 promotes phagocytosisinduced neutrophil apoptosis and accelerates resolution of pulmonary 
inflammation. Proceedings of the National Academy of Sciences of the United States of America 109, 14983-8 (2012).

236. Martin, M.C., Dransfield, I., Haslett, C. \& Rossi, A.G. Cyclic AMP regulation of neutrophil apoptosis occurs via a novel protein kinase A-independent signaling pathway. J Biol Chem 276, 45041-50 (2001).

237. Godson, C. et al. Cutting edge: lipoxins rapidly stimulate nonphlogistic phagocytosis of apoptotic neutrophils by monocyte-derived macrophages. $J$ Immunol 164, 1663-7 (2000).

238. Bystrom, J. et al. Resolution-phase macrophages possess a unique inflammatory and bactericidal phenotype that is controlled by cAMP. Blood. 112 (2008).

239. Sun, L. et al. Tristetraprolin (TTP)-14-3-3 complex formation protects TTP from dephosphorylation by protein phosphatase $2 a$ and stabilizes tumor necrosis factor-alpha mRNA. J Biol Chem 282, 3766-77 (2007).

240. Fredman, G., Van Dyke, T.E. \& Serhan, C.N. Resolvin E1 regulates adenosine diphosphate activation of human platelets. Arterioscler Thromb Vasc Biol 30, 2005-13 (2010).

241. Rajakariar, R. et al. Nonresolving inflammation in gp91phox-/- mice, a model of human chronic granulomatous disease, has lower adenosine and cyclic adenosine 5'-monophosphate. J Immunol 182, 3262-9 (2009). 